\title{
Mapping a Complete Neural Population in the Retina
}

\author{
Olivier Marre, ${ }^{1 *}$ Dario Amodei, ${ }^{1 *}$ Nikhil Deshmukh, ${ }^{1}$ Kolia Sadeghi, ${ }^{1,3}$ Frederick Soo, ${ }^{1}$ Timothy E. Holy, ${ }^{4}$ \\ and Michael J. Berry II ${ }^{1,2}$ \\ ${ }^{1}$ Department of Molecular Biology and ${ }^{2}$ Princeton Neuroscience Institute, Princeton, New Jersey 08544, ${ }^{3}$ Center for Theoretical Neuroscience, Columbia \\ University, New York, New York 10027, and ${ }^{4}$ Department of Anatomy and Neurobiology, Washington University in St. Louis, St. Louis, Missouri 63130
}

Recording simultaneously from essentially all of the relevant neurons in a local circuit is crucial to understand how they collectively represent information. Here we show that the combination of a large, dense multielectrode array and a novel, mostly automated spikesorting algorithm allowed us to record simultaneously from a highly overlapping population of $>200$ ganglion cells in the salamander retina. By combining these methods with labeling and imaging, we showed that up to $95 \%$ of the ganglion cells over the area of the array were recorded. By measuring the coverage of visual space by the receptive fields of the recorded cells, we concluded that our technique captured a neural population that forms an essentially complete representation of a region of visual space. This completeness allowed us to determine the spatial layout of different cell types as well as identify a novel group of ganglion cells that responded reliably to a set of naturalistic and artificial stimuli but had no measurable receptive field. Thus, our method allows unprecedented access to the complete neural representation of visual information, a crucial step for the understanding of population coding in sensory systems.

\section{Introduction}

Throughout the brain, local circuits represent information using large populations of neurons. By recording neurons one at a time, one can develop an overall picture of how a neural population divides its encoding task up among its many different neurons (Hubel and Wiesel, 1962; Hahnloser et al., 2002). However, many properties of this division of labor are idiosyncratic, such as the tiling of receptive fields (Wässle and Boycott, 1991) or the boundaries of cortical columns (Ohki et al., 2005), and can only be witnessed in groups of cells recorded simultaneously. Because the structure of the neural code depends very sensitively on the correlations among neurons (Averbeck et al., 2006; Schneidman et al., 2006), one must record simultaneously from essentially all of the relevant neurons- $\mathrm{a}$ feat that has never before been accomplished at the level of individual spikes - to understand how they collectively represent information.

The vertebrate retina is an excellent system for recording from large and complete neural populations. Planar multielectrode arrays can record simultaneously from many ganglion cells (Meister et al., 1994). Furthermore, the retina is organized into precise spatial modules, so that a small location in visual space maps onto a single subset of the entire retina. How big is this area? In the

Received Feb. 15, 2012; revised Aug. 8, 2012; accepted Aug. 13, 2012.

Author contributions: 0.M., D.A., K.S., T.E.H., and M.J.B. designed research; 0.M., D.A., N.D., F.S., T.E.H., and M.J.B. performed research; 0.M., K.S., T.E.H., and M.J.B. analyzed data; 0.M., D.A., T.E.H., and M.J.B. wrote the paper.

This work was supported by National Institutes of Health/National Eye Institute Grants EY 014196 and EY 017934 (M.J.B.), National Science Foundation Grant IIS-0613435 (M.J.B.), National Institutes of Health/National Institute on Deafness and Other Communication Disorders Grant DC 005964 (T.E.H.), and the Hertz Foundation (D.A.). We thank William Bialek, Stephanie Palmer, Phil Nelson, Vijay Balasubramanian, and Marcelo Magnasco for helpful discussions and Benjamin Singer for excellent technical assistance with the Princeton computing cluster.

* $0 . M$. and D.A. contributed equally to this work.

Correspondence should be addressed to Olivier Marre at his present address: Institut de la Vision, 17 rue Moreau, 75012 Paris, France. E-mail: olivier.marre@inserm.fr.

DOI:10.1523/JNEUROSCI.0723-12.2012

Copyright $\odot 2012$ the authors $\quad 0270-6474 / 12 / 3214859-15 \$ 15.00 / 0$ salamander, ganglion cell pairs possess significant redundancy in the visual information they encode when they are separated by up to $200 \mu \mathrm{m}$ (Puchalla et al., 2005). A circle of this radius contains $\sim 200$ ganglion cells, which defines the extent of the population that collectively encodes overlapping visual information.

What is needed, therefore, is a large multielectrode array, dense enough to record from all the cells over the array and large enough to cover the spatial extent over which information is redundant. In addition, datasets of this size necessitate a spikesorting algorithm that is extensively automated. Finally, because multiple nearby ganglion cells often fire together, the spikesorting algorithm must work with overlapping spike waveforms. Large-scale multielectrode recordings have been performed previously (Frechette et al., 2005), but in these data, only one or two cell types were sampled completely. In addition, the spike-sorting algorithm did not explicitly model spike overlaps. Dense, complete recordings have been performed previously (Segev et al., 2004), but these data were taken with an array too small to sample the complete population and used an algorithm that does not scale up to larger datasets.

Here we report the design and fabrication of a recording device with electrodes densely covering an area matching the spatial range of redundancy between ganglion cells and a novel, semiautomated spike-sorting algorithm suited for the recordings. By combining these methods with labeling and imaging of the ganglion cells, we demonstrate that we recorded from up to $95 \%$ of the ganglion cells over the array. We found a region of visual space for which we recorded almost all the cells whose receptive field overlapped with this area, indicating a nearly complete coverage of this region by our recordings.

We clustered the ganglion cells into seven functional types based on their receptive field dynamics and found that only one cell type tiled visual space. We also discovered a subset of ganglion cells with weak receptive fields that nonetheless responded precisely and reliably to a set of artificial and natural stimuli. 


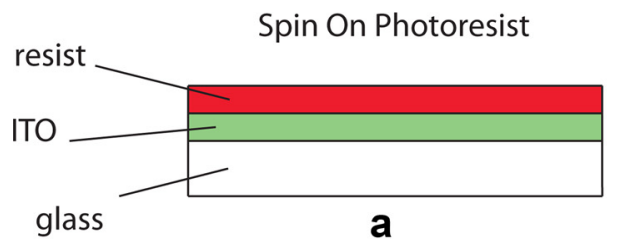

RCA2/Acetone clean

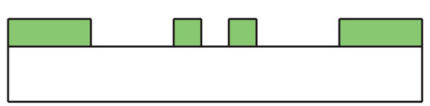

d Pattern Insulator Holes

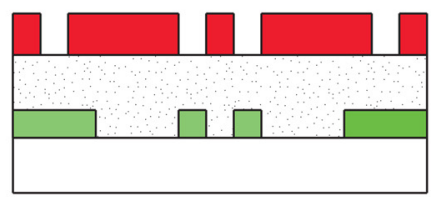

g
Pattern Photoresist

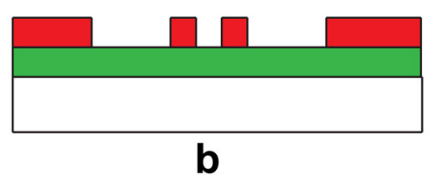

Deposit Insulator

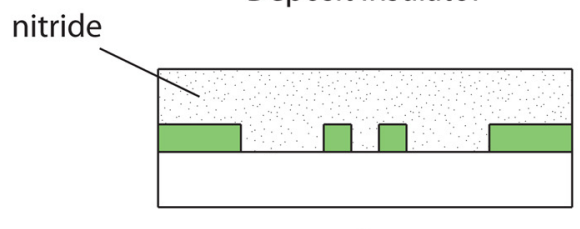

e

Etch Insulator Holes (SF6)

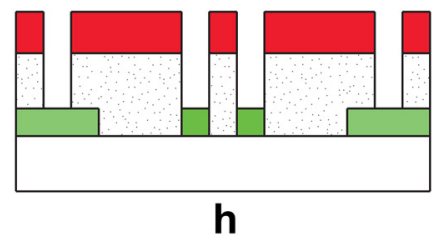

Platinization

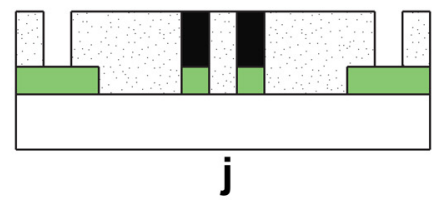

Etch ITO: Argon Sputter

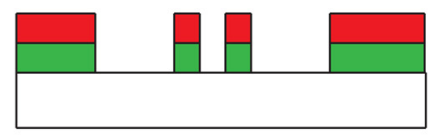

C

Spin On Photoresist

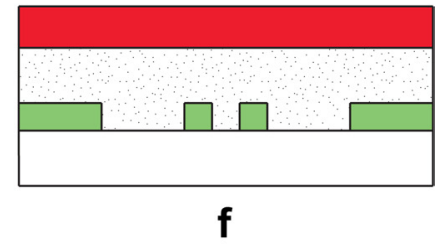

Acetone Clean

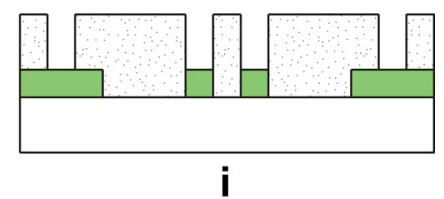

Figure 1. $\quad \boldsymbol{a}, \boldsymbol{b}$, Photolithographic patterning of both large and small features. $\boldsymbol{c}$, Argon sputter to etch ITO. $\boldsymbol{d}$, Cleaning via RCA2 followed by acetone sonication to remove resist hardened from sputtering. $\boldsymbol{e}$, Two-stage deposition of silicon nitride insulator. $\boldsymbol{f}-\boldsymbol{i}$, Etching of holes in the insulator to form electrodes and readout pads. $\boldsymbol{j}$, Platinization to increase the surface area and thus the conductivity of the electrodes.

\section{Materials and Methods}

Array fabrication. To achieve our objectives of densely sampling the electrical activity of a complete population of retinal ganglion cells, we designed multielectrode arrays to have 252 electrodes. Electrodes were arranged on a square lattice with $30 \mu \mathrm{m}$ spacing, because that design was successful for 30 electrode arrays (Segev et al., 2004). To achieve this spacing with 252 electrodes, we designed electrodes to have a $6 \mu \mathrm{m}$ diameter and wires to have a width of $2.0 \mu \mathrm{m}$ (Fig. 1). This is well above the minimum feature size that can be produced with photolithography, allowing the entire array to be patterned with a single photolithographic mask in an ordinary mask aligner, in contrast to previous arrays that required multiple masks and/or electron beam lithography (Litke et al., 2004).

We begin with a 4 -inch quartz wafer coated with $250 \mathrm{~nm}$ of indium tin oxide (ITO) (Bay View Optics). The ITO had a resistance of $10-15 \mathrm{Ohm} /$ square and an optical transmittance of $95 \%$ in the visible range. The arrays were first cleaned in RCA1 clean (Radio Corporation of Americadeveloped cleaning solution, containing 5:1:1 $\mathrm{H}_{2} \mathrm{O} / \mathrm{H}_{2} \mathrm{O}_{2} / \mathrm{NH}_{4} \mathrm{OH}$ ) for $10 \mathrm{~min}$, followed by acetone for $10 \mathrm{~min}$. A $1.2 \mu \mathrm{m}$ layer of Megaposit SPR 3617 dyed photoresist (Shipley) was spun on and exposed and developed with a single photomask containing the electrodes, leads, bond pads, and four ground wires of the array. After a $60 \mathrm{~s}$ hard bake $\left(110^{\circ} \mathrm{C}\right.$ hotplate bake), the ITO was etched via argon-ion sputtering in a reactive ion etcher (RIE), using the photoresist as a mask. The etch was performed in an RIE (MRC model 55) at $100 \mathrm{~W}$ power, 10 mtorr pressure, and 10 standard cubic centimeters $\left(\mathrm{scm}^{3}\right)$ flow rate of argon, for $22 \mathrm{~min}$. Next, the resist was stripped by a $20 \mathrm{~min}$ RCA1 clean, followed by 3 min of sonication in acetone. This procedure was effective despite partial burning of the photoresist during the argon sputter.

Next, the array was coated with an insulator to electrically isolate the channels from one another and from the bath into which the array will be immersed. To accomplish this, a 1- $\mu$ m-thick silicon nitride layer was deposited via alternating frequency $(13.67 \mathrm{MHz}$ and $400 \mathrm{kHz}$, alternation cycle of 2 and $5 \mathrm{~s}$ ) plasma enhanced chemical vapor deposition at $350^{\circ} \mathrm{C}$ (Surface Technology Systems). The deposition took place in two layers of $500 \mathrm{~nm}$ each to minimize the density of pores in the insulator. To make the array functional, holes were then etched in the insulator to expose the recording electrodes, the ground electrodes, and the readout pads on the edge of the array. For this, a $1 \mu \mathrm{m}$ layer of Megaposit SPR 3612 photoresist (Shipley) was spun on and patterned, and the insulator was then etched via RIE (MRC model 55) with $15 \mathrm{scm}^{3}$ trifluoromethane and 3 $\mathrm{scm}^{3} \mathrm{O}_{2}$, at $50 \mathrm{~W}$ and $50 \mathrm{~m}$ torr for $30 \mathrm{~min}$. Finally, the electrodes were platinized one by one via application of a $4 \mathrm{~V}$ direct current potential to a platinum wire in a chloroplatinic acid solution for 10-60 s. A completed array is shown in Figure $2 A-C$. To give accurate measurements of the extracellular voltage, the array must have relatively low input impedance with relatively low amounts of dispersion and also low noise and leakage current. To understand the electrical behavior of the array, we used a standard model (Litke et al., 2004) for resistance in recording electrodes.

Briefly, the model includes the resistance of the ITO wires leading to the electrodes, followed in series by the electrode impedance (which consists of both a capacitive polarization component and a resistive charge transfer component), followed by the "spreading resistance," the resistance attributable to the bottleneck of current spreading out from the (small) electrode surface into the extracellular medium. For our array, the wires are predicted to have a direct current impedance of 5-10 $\mathrm{k} \Omega$. The resistive electrode impedance is given by $R_{e}=1 /\left(\sigma_{e} A\right)$, where $\sigma_{e}$ is a surface ion conductivity and $A$ the interface area, and the capacitive electrode impedance is given by $C_{e}=\varepsilon_{0} k A / d$, where $d$ is the electrode surface charge separation distance, $k$ is the dielectric constant of the 
A

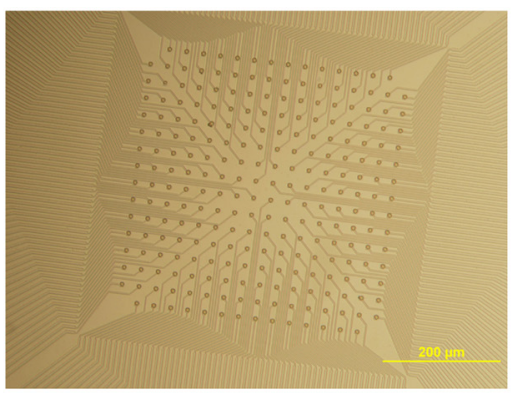

B

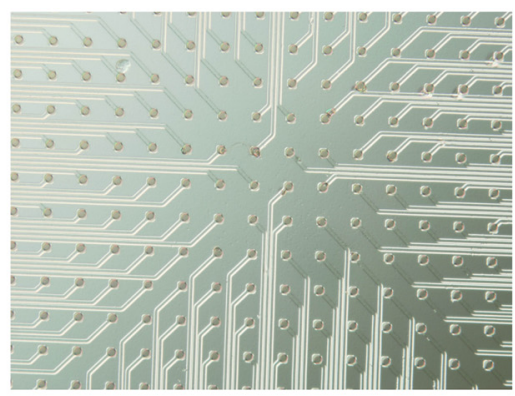

C

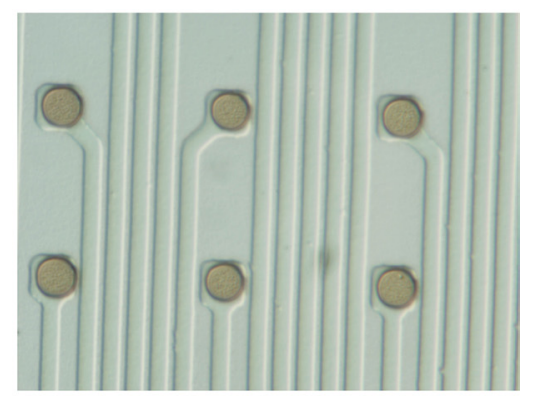

D

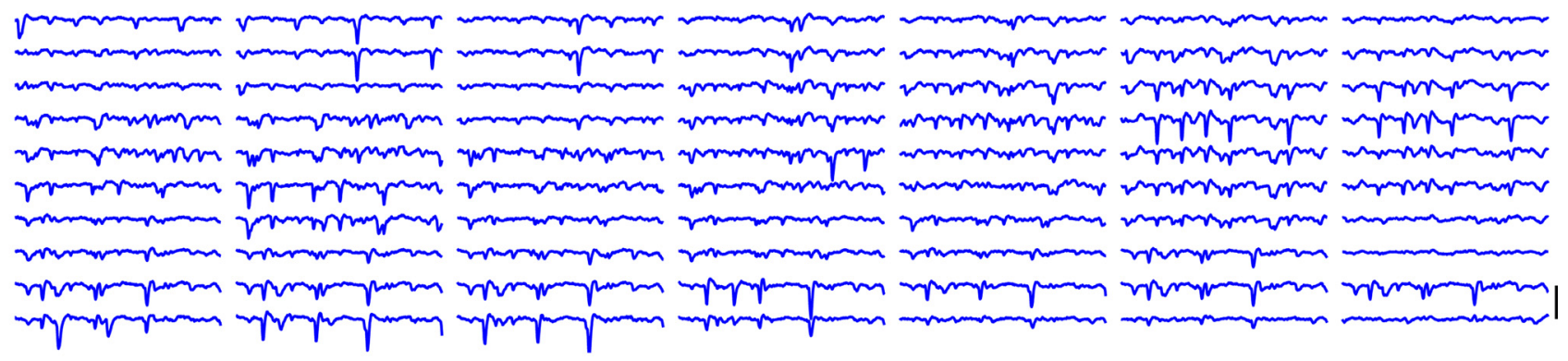

E

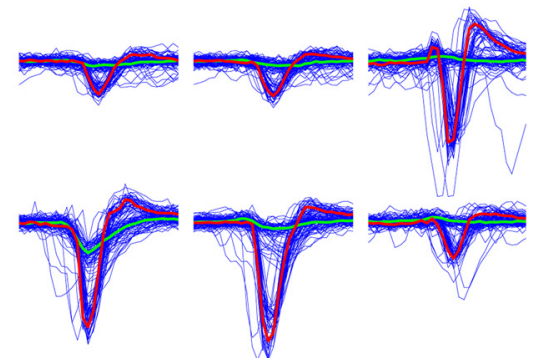

F

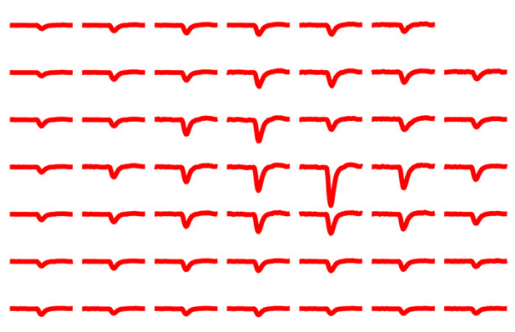

G

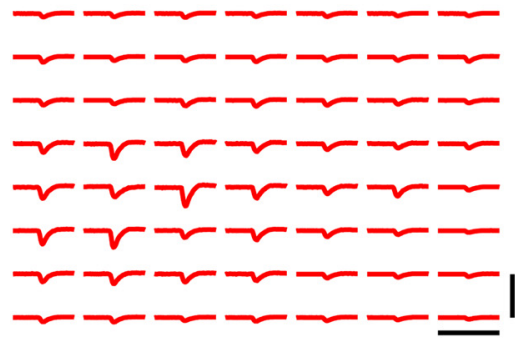

Figure 2. $A-C$, An example of a dense multielectrode array with 252 electrodes, shown at three different magnifications; the distance between electrodes is $30 \mu \mathrm{m}$. $\boldsymbol{D}$, Example of raw data recorded from a retina pressed against the array. Each trace represents the voltage measured on one electrode. Here we represent traces from 70 electrodes. Temporal window size, $40 \mathrm{~ms}$. Calibration bar, $400 \mu \mathrm{V}$. $\boldsymbol{E}$, Superimposed snippets (blue) of the recording that correspond to two different cells, whose templates are indicated in red and green. $\boldsymbol{F}, \boldsymbol{G}$, Two examples of templates extracted by the algorithm. Calibration: $6.5 \mathrm{~ms}, 200 \mu \mathrm{V}$.

interface, and $A$ is the interface area. For saline, $k \approx 80$ and $d \approx 0.5 \mathrm{~nm}$. The area $A$ is not the geometrical area of the electrode but is $\sim 100$ times larger because of the granularity of the platinum black. For our arrays, we estimate an electrode capacitance of $C_{e}=5-10 \mathrm{nF}$ in parallel with a resistance of $R_{e}=\sim 1 \mathrm{M} \Omega$ (hard to measure precisely because it has to be measured at near direct current), giving a total electrode impedance ranging from $Z_{e}=200-300 \mathrm{k} \Omega$ at $100 \mathrm{~Hz}$ to $Z_{e}=90 \mathrm{k} \Omega$ at $10 \mathrm{kHz}$.

The spreading resistance is given by $R_{s}=\rho / 4 r$, where $r$ is the electrode radius, and $\rho$ is the conductivity of the medium (Borkholder, 1998). For our array, $\rho=0.72 \Omega$-m for saline medium, $r=3 \mu \mathrm{m}$, and thus $R_{s} \approx 80$ $\mathrm{k} \Omega$. Overall, we predict an impedance ranging from $Z_{\text {tot }}=200 \mathrm{k} \Omega$ at 100 $\mathrm{Hz}$ and to $Z_{\text {tot }}=90 \mathrm{k} \Omega$ at $10 \mathrm{kHz}$. From the impedance, we can derive a thermal noise $\delta_{V}^{2}=4 k_{B} T Z_{\text {tot }}(\omega)$, where $T$ is temperature, $k_{\mathrm{B}}$ is Boltzmann's constant, and $Z_{\text {tot }}(\omega)$ is the frequency-dependent impedance; integrated over a $10 \mathrm{kHz}$ bandwidth, this gives a 5-10 $\mu \mathrm{V}$ thermal noise. The amplifier emits an additional noise of 5-10 $\mu \mathrm{V}$ according to the specifications of the manufacturer (Multi-Channel Systems). We compared this model with experimental measurements of the alternating current impedance of each channel while the array was immersed in Ringer's medium. At the frequencies of interest for recording neural spikes ( $100 \mathrm{~Hz}$ to $10 \mathrm{kHz}$ ), the measured impedance ranged from 50 to $300 \mathrm{k} \Omega$, which fits well with the theoretical model. We measured a noise range of $5-15 \mu \mathrm{V}$ (average of $6 \mu \mathrm{V}$ ), which fits well with the model.

Recordings. Experiments were performed on the larval tiger salamander (Ambystoma tigrinum tigrinum) of either sex, in accordance with institutional animal care standards. Retinas were isolated from the eye in darkness (Puchalla et al., 2005); the retina was detached and the pigment epithelium removed. The photoreceptor side was stuck to a semipermeable membrane previously bathed in poly-L-lysine and attached on an aluminum ring. The ring was attached to a gantry and pressed against the array in a controlled manner. The retina was superfused with oxygenated Ringer's medium $\left(95 \% \mathrm{O}_{2}, 5 \% \mathrm{CO}_{2}\right)$ at room temperature $\left(22^{\circ} \mathrm{C}\right)$. Extracellular recordings were acquired and digitized by a 252 channel preamplifier (Multi-Channel Systems) and stored for offline analysis. Five animals were used for these experiments.

Preprocessing. The extracellular recordings were composed of fast, mostly negative voltage fluctuations, reflecting the spiking activity of nearby ganglion cells, riding on slow fluctuations that corresponded to the average electrical potential contributed by all of the major cell types in the retina and believed to be dominated by the activity of bipolar cells. Our first aim was to remove these low-frequency fluctuations present in the recordings, while not altering the spike waveforms. On each channel, we first did a crude detection of the spike times on a high-pass filtered version of the voltage signal. Then, the spikes were removed from the original voltage signal and replaced by a linear interpolation of the slow trend in the voltage. The slow fluctuations were then estimated on the resulting signal by a second-order polynomial interpolation over a sliding window (typically $100 \mathrm{~ms}$ ). The low-frequency fluctuations estimated this way were then subtracted from the original voltage trace. Compared with high-pass filtering, the advantage of this technique was that we isolated and removed the slow fluctuations without affecting the detailed temporal waveform of action potentials. This is important, because the subtraction process varies from one instance of the spike of a 
cell to another, so introducing additional shape variation would reduce the quality of the subsequent spike sorting.

Next, we performed spatial whitening. Using our rough detection of spikes, we found periods of silence over the entire array. Using these periods, we estimated the spatial covariance matrix of the noise, i.e., $N_{i j}=$ $\left\langle s_{i}(t) s_{j}(t)\right\rangle$. We then took the square root of the inverse covariance matrix, $M$, and multiplied the voltage signal $\mathbf{s}(t)$ by this matrix. This put the raw voltage signals into normalized units of signal-to-noise (SNR) ratio (Prentice et al., 2011). For some figures, we restored physical units by multiplying the normalized signal by the root-mean-squared value of the noise on a given electrode.

Spike sorting. We decomposed the recorded voltages into spikes attributed to different cells according to the following model described in the results (Eq. 3 below). In this model, the raw signal recorded over 252 electrodes, $\mathbf{s}(t)$, is decomposed into a sum of templates and a noise term, $\mathbf{e}(t)$. Each template $\mathbf{w}_{j}(\tau)$ corresponds to a different cell $j$ and reflects the average spatiotemporal waveform triggered on the electrodes when cell $j$ fires an action potential. A template is added to this sum at time $t_{i}$ when the cell spikes and is weighted by an amplitude factor $a_{i j}$ that varies for each spike time $t_{i}$.

Our sorting algorithm aimed at finding the unknown parameters of this model. These parameters are of two kinds: the templates $\mathbf{w}_{j}(\tau)$ and the amplitudes $a_{i j}$. Note that most of the amplitude values should be equal to 0 , because only a small fraction of all the neurons will fire at any particular time. Our sorting can mostly be decomposed in two steps: (1) we cluster spikes and estimate their template; and (2) we fit the set of templates to the raw data to obtain spike times for each ganglion cell. These core steps are described in Results.

In principle, the spikes we recorded could come from displaced amacrine present in the ganglion cell layer. However, this possibility is made very unlikely by the size of the spikes of amacrine cells. These spikes are small $(\sim 20 \mathrm{mV})$, even in intracellular recordings (Barnes and Werblin, 1986; Sakai et al., 1997; Miller et al., 2006). If the spike of the amacrine cell is five times smaller than that of the ganglion cell, then it often will not exceed our spike threshold in the preprocessing step. Furthermore, for spikes that barely exceed the threshold, templates with a maximum amplitude close to the noise level typically do not pass our test for a clean refractory period. So, in the following, we assume that the cells we recorded from were ganglion cells.

Clustering. We began by extracting a set of candidate spikes from the raw data. The candidate spikes were detected by flagging all local minima in the preprocessed data on any of the 252 channels that exceeded a threshold of six times the SD. These spike times were noted $\left\{t_{i}\right\}$. To cluster them into groups corresponding to different cells, we represented each flagged spike event $t_{i}$ by the vector of voltage values on all 252 electrodes at that time, $\mathbf{s}\left(t_{i}\right)$. Each such vector is referred to as a "data point" in a 252-dimensional space. These were first divided into groups $\left\{\mathbf{s}\left(t_{i}^{k}\right)\right\}$ according to which electrode $k$ had the largest voltage for a given spike. Within each group, we then clustered the vectors using an algorithm based on mean shift clustering. The idea of this algorithm is that each vector can be represented as a point in a 252-dimensional space. The clusters correspond to regions of this space in which the density of points is maximal.

Mean shift clustering is a nonparametric technique that groups points sharing a common density "peak" (Fukunaga and Hostetler, 1975; Comaniciu and Meer, 2002). A set of probe points flow to their local peak by repeatedly moving to the center of mass of all data points (which are fixed) within a radius $R$. For our algorithm, probe point starting positions were chosen from all of the real data points that we measured. To ensure coverage of all data points, the $k$ th probe point was chosen iteratively as the data point most distant from the first $k-1$ probe points. For $m$ probe points and $N$ data points (spikes), this is an operation of $O(m N)$; we used $m=1000$. Specifically, for a given probe point at position $\mathbf{x}_{\mathbf{0}}$, we determined the $n$ points $\left\{\mathbf{x}_{\mathbf{i}}\right\}$ which are within a radius $R$ from $\mathbf{x}_{\mathbf{0}}$ (i.e., $\left|\mathbf{x}_{\mathbf{0}}-\mathbf{x}_{\mathbf{i}}\right| \leq R$ ). We computed the mean-shift vector $\Delta \mathbf{x}=\frac{1}{n} \Sigma_{i}\left(x_{i}-\mathbf{x}_{0}\right)$. The position of the probe point is then updated: $\mathbf{x}_{\mathbf{0}}=\mathbf{x}_{\mathbf{0}}+\Delta \mathbf{x}$. This shift was iterated until it reached a local maxima of the density. Our algorithm differed from conventional mean shift by adjusting $R$ at each step of the flow to achieve statistical confidence in the location of the center of mass (T.E.H., unpublished observation).

Note that, because the spike times are grouped according to the electrode in which the voltage peaked, a single cell evoking similar voltage deflection on two electrodes would be split into two clusters. More generally with our technique, it is possible that the waveforms corresponding to a single cell could be split into more than one cluster, because, for example, of the variability of the waveform amplitude or because the ganglion cell fired bursts of spikes in which the first spike was larger than the rest (Segev et al., 2004). In these cases, two or more templates were initially produced, but they were later merged together, as explained in the section below.

Template extraction. We defined the template for a cluster $j$ as the point-wise median of all the spike waveforms in the cluster, in which the spike waveform for an event time $t_{i}$ consists of a $6.5 \mathrm{~ms}$ sample on all channels centered on $t_{i}$ (examples in Fig. $2 F, G$ ). Notice that, although our clustering procedure used 252-dimensional data point, each template has $252 \times 65$ components. We also defined the normalized template, $\hat{\mathbf{w}}_{j}(t)=\frac{\mathbf{w}_{j}(t)}{\left|\mathbf{w}_{j}(t)\right|}$.

Fitting the templates. We next use the templates to fit the raw data using the form of Equation 3. Because the templates, $\mathbf{w}_{j}(\tau)$, have been defined before this stage of the algorithm, our goal in this step was to find the values of the amplitudes $a_{i j}$. For that purpose, we used an iterative algorithm that is described in Results (see Fitting the templates). For each cell, two parameters defined the range of acceptable values for the amplitude: $a_{j}^{\min }$ and $a_{j}^{\max }$.

Extracting all the templates. After fitting, we used a second run of the clustering algorithm to separate out additional cells. We focused on trying to distinguish cells that were erroneously clustered together in the first run. For each template, we took all the spikes assigned to it by the fitting algorithm and reran the mean-shift clustering on just a subset of all of the spikes. We extracted templates from all of these clusters and reran our fitting procedure, as described above, with the new, expanded set of templates.

Up until this point, our method has been fully automated. We next used a couple of additional steps done by hand to adjust and improve the results. First, we manually inspected each template for the possible presence of multiple cells by visually superimposing all the spike waveforms that were assigned to a given cell (Fig. $2 E$ ). If two clearly delineated groups of spikes could be discriminated based on obvious differences in shape, we then split the cluster into two new clusters. This splitting was done by manually grouping together the waveforms corresponding to the same shape. Such manual intervention was only necessary for a small fraction of the templates (typically $20 \%$ ). This manual intervention was made necessary because some templates were best discriminated using not only the peak voltages but also the full shape of the waveform. The number of cases in which manual intervention was necessary might thus be reduced by feeding the clustering algorithm with the results of the projection of each snippet onto some well-chosen vectors (Quiroga et al., 2004). Having extracted these new clusters, we reran the fitting process on the raw data.

Adjustment of the amplitude thresholds by hand was sometimes necessary in the case in which two cells had templates very similar in shape but differing primarily in amplitude. We selected the proper amplitude thresholds by plotting the histogram of amplitudes for both cells and choosing the minimum separating the two peaks. For long recordings, some templates changed their mean amplitude $a$ over time. For these cells, we had to choose amplitude thresholds that would also vary with time. We estimated the median amplitude $\bar{a}(t)$ in time slices that were long enough ( $>5 \mathrm{~min}$ ) to allow a reliable estimation of this "local mean." This estimation of the mean amplitude over time was adjusted by hand in a few cases. The amplitude thresholds at time $t$ were then defined as a rescaled version of $\bar{a}(t): a_{j}^{\min }(t)=a_{j}^{\min } \bar{a}(t)$ and similarly for $a_{j}^{\max }$. Using these time-dependent thresholds allowed us to cope with slow changes of spike amplitude over hours of recordings.

Merging duplicate templates. Having discovered a large number of putative templates, we next turned our attention to merging duplicate templates (pairs of templates that actually represent the same cell, as 
mentioned above). Duplicate templates arise for two primary reasons: (1) some ganglion cells fire in short bursts of spikes in which the first spike is often larger than the subsequent spikes; and (2) some cells have more highly variable spike amplitudes, and our clustering algorithm splits this continuum into two or more clusters. Such duplicate template pairs were easily identifiable using criteria described in previous studies (Harris et al., 2000; Segev et al., 2004). Duplicate templates have very similar shapes, identical receptive fields, few or no refractory period violations when their spike trains are superimposed, and a cross-correlogram with a large dip near a time lag of zero attributable to the fact that a single cell cannot spike twice in a short interval (typically $<2 \mathrm{~ms}$ ). We combined such spike trains into a single cell.

Note that, although this step was performed manually, we compared only the pairs of templates above a criterion similarity, defined as a normalized cross-correlation above 0.75 . For a single template, we rarely found more than five pairs above that threshold, so this manual step remained linear with the number of templates.

We also checked for the presence of axonal spikes, which can be identified by a characteristic triphasic potential. A few such spikes were found, and we excluded such axonal templates because the tight bundling of multiple axons makes it unlikely that their waveforms were properly separated. As a final check that no more templates contained spikes from multiple cells, we counted the number of refractory period violations for each sorted cell. Half of the cells showed no refractory period violations, and $79 \%$ of the cells showed $<0.1 \%$ of them (see Fig. $4 D$ ), indicating that very few such errors remained.

Quantifying goodness of fit. To quantify the goodness of the fit of our model (templates and amplitudes) to the raw data, we had to take into account that the percentage of variance explained by the model will always be limited by the background noise present in the recordings [the variable $\mathbf{e}(t)$ in Eq. 3]. So we modified the standard calculation of the percentage of variance explained by our model to discount for the variance cause by noise:

$$
R^{2}=1-\frac{V_{\text {residual }}-V_{\text {noise }}}{V_{\text {total }}}
$$

where $V_{\text {residual }}$ is the variance of the residual (voltage - prediction), $V_{\text {noise }}$ is the variance of the background noise, estimated on segments of the raw electrical recordings that contained no spikes, and $V_{\text {total }}$ is the total variance of the data.

Quantifying cell separation. Many studies have quantified the degree of separation between cells, by identifying the degree of overlap between their waveforms. Although we believe that our surrogate data tests (see Results, Fitting performance and correlation estimation) provide a better estimate of spike-sorting errors, we performed with calculation as an additional check on our method. We used a method similar that used previously (Pouzat et al., 2002; Rinberg et al., 2003; Hill et al., 2011) to estimate the mixing probability between the different clusters of spike waveforms. The idea behind this method is to take the full spatiotemporal waveforms triggered by each cell and to estimate whether a waveform associated with one cell could have been mistaken as belonging to the other cell. An example of such a superposition of waveforms belonging to two different cells is shown in Figure $2 E$. To estimate the mixing probability, the voltage waveforms associated with each cell were fitted with a multivariate Gaussian distribution. Then, for each pair of cells, we estimated a distance between these two distributions as defined previously (Hill et al., 2011).

A single spatiotemporal waveform can be described as a vector with $252 \times 65$ coordinates, in which 252 is the number of electrodes and 65 the size of the temporal window. We thus describe the $i$ th waveform of cell $j$ as a vector $\mathbf{v}_{i, j}$ with $252 \times 65$ coordinates and model it as follows:

$$
\mathbf{v}_{i, j}=\mathbf{m}_{j}+\mathbf{g}_{\mathbf{i}, \mathbf{j}},
$$

where $\mathbf{m}_{j}$ is the mean of the waveforms and $\mathbf{g}_{\mathbf{i}, \mathbf{j}}$ is a Gaussian noise with mean 0 and covariance $G_{j}$.

The main challenge was to estimate this covariance matrix, which has, in principle, $(252 \times 65)^{2}$ coefficients. To reduce the number of parameters in this covariance matrix, we always restricted ourselves to elec- trodes in which the template peaked at a value of at least $10 \%$ of its maximum. Then, because the signal had been previously whitened between electrodes (see above), we made the simplifying assumption that the residuals from each electrode were independent and that they had the same temporal covariance. This led us to estimate only $65^{2} / 2$ coefficients of the covariance matrix. The estimation of the covariance matrix could also be strongly biased by the spikes from other cells that would overlap with the waveforms we used for the covariance matrix estimation. To suppress this potential bias, for each waveform, we subtracted all the other templates that had been fitted to the data in close vicinity.

Once we had estimated the covariance matrix, this model allowed computation of the $\chi^{2}$ error of how well this model accounted for the voltage waveforms measured for each cell (Hill et al., 2011). The average $\chi^{2}$ value (divided by the number of dimensions of $\mathbf{g}_{\mathbf{i}, \mathbf{j}}$ ) was $1.3 \pm 0.3(n=$ 224 cells). The deviation from the theoretical value of 1 measures the degree to which the assumption of Gaussian noise is not valid, as has been reported previously (Fee et al., 1996).

For each pair of cells A and B, we estimated the average likelihood that each spike waveform of cell A could be generated by template B, according the previously fitted model. This probability that the $i$ th waveform of cell $j$ could be generated by cell $k$ is then: $P\left(\mathbf{v}_{i, j} \mid k\right)=(2 \pi)^{-0.5 \times d} G_{k}^{-0.5} \exp ^{-0.5\left(\mathbf{v}_{i, j}-\mathbf{m}_{k}\right) G_{k}^{-1}\left(\mathbf{v}_{i, j}-\mathbf{m}_{k}\right)}$, where $d$ is the number of dimensions, $252 \times 65$ (Hill et al., 2011). We then computed the probability that the spike waveform could be mistaken for cell B using a Bayesian inversion, similar to that used previously (Hill et al., 2011).

Computational cost. Even if this algorithm is demanding in terms of computing resources, the computational cost remained a linear function of the number of electrodes and of the number of spike times. The clustering can be done independently for the different electrodes, the template extraction can be done independently for the different clusters, and the fitting part, as well as the initial spike detection, can be done on different parts of the recording independently.

To take advantage of this potential, we parallelized all the steps of the algorithm and split each step into many (usually 200) tasks, each taking a different part of the workload, that we ran in parallel on a computing cluster. The cluster consisted of 13 units, for a total of 52 nodes. Each node contained two quad-core Xeon X5570 processors (Intel), operating at $2.7 \mathrm{GHz}$, for a total of 416 processors, and $24 \mathrm{~GB}$ of RAM for a total of 1.25 TB of RAM.

Although the total running time varied from experiment to experiment, most runs were completed overnight.

Labeling. We labeled ganglion cells with the rhodamine dextran in a manner similar to that used previously (Segev et al., 2004; Behrend et al., 2009). Briefly, salamander eyes were removed and placed in Ringer's solution, leaving an optical nerve stump. A crystal of rhodamine dextran dye coupled with biotin (Invitrogen) was placed on the stump, and a collar was glued around it to avoid unwanted dilution of the dye. The eyecup was then incubated for 2-4 h to let the dye actively transport along the optic nerve and invade ganglion cells before recording. The cells were imaged by epifluorescence after the recording.

Visual stimulation. To measure receptive fields, a black and white checkerboard was displayed to the retina. The size of each check was 69 $\mu \mathrm{m}$, and each check was picked randomly as black or white 30 times per second. The repeated natural movie was a movie of a fish tank captured at $30 \mathrm{~Hz}$ with a standard camera. It lasted $20 \mathrm{~s}$. The moving bar was a dark bar on a gray background with a width of $150 \mu \mathrm{m}$ moving at a speed of $0.52 \mathrm{~mm} / \mathrm{s}$. The moving gratings spanned a range of speeds from 0.52 to $1.82 \mathrm{~mm} / \mathrm{s}$ and spatial frequencies from 1 to 7 cycles $/ \mathrm{mm}$.

\section{Results}

\section{Array fabrication and recordings}

We fabricated dense, transparent multielectrode arrays with 252 electrodes arranged on a square lattice (Fig. $2 A-C$ ). The density of the electrodes (30 $\mu \mathrm{m}$ spacing) corresponded to the decay length of extracellular signals (Segev et al., 2004), and the total dimension of the array, $450 \times 450 \mu \mathrm{m}$, matched the spatial extent of redundancy between ganglion cells (Puchalla et al., 2005). Arrays were made of ITO, a transparent semiconduc- 


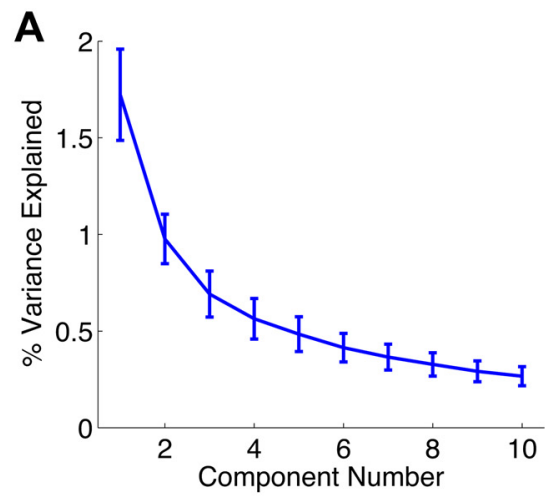

D

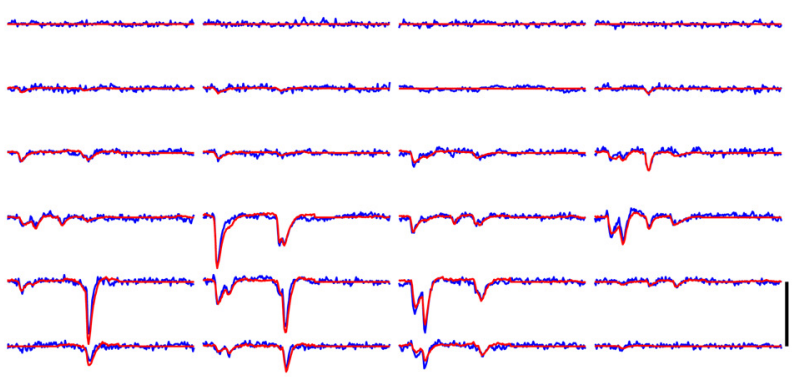

$\mathbf{B}_{1}$

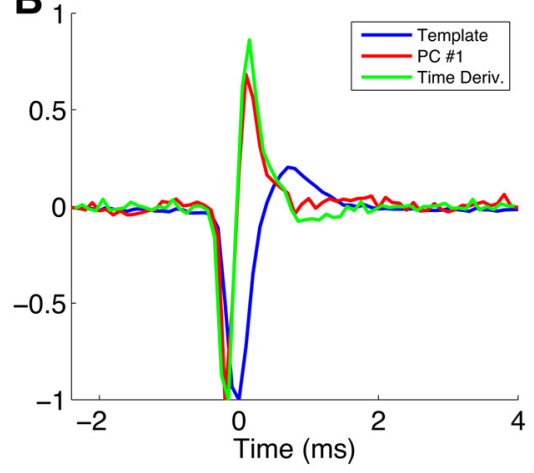

C

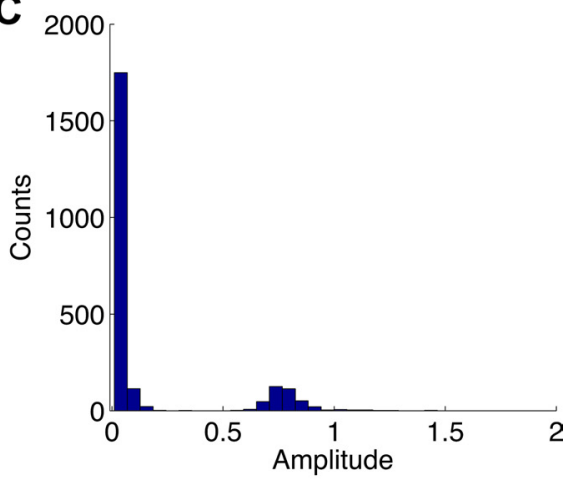

E

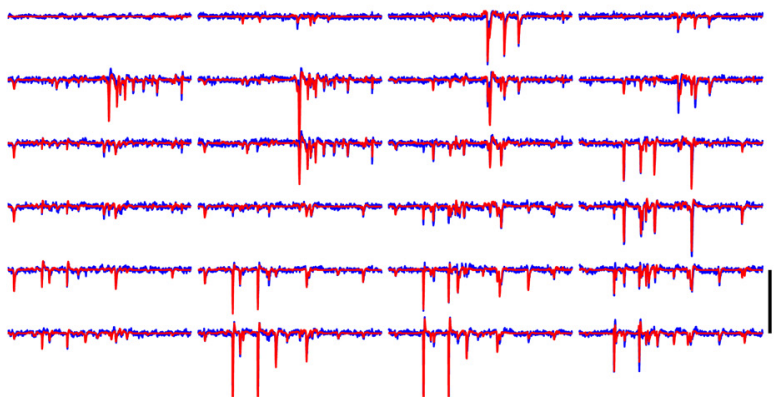

Figure 3. A, Percentage of the voltage variability explained by the different principal components extracted from the analysis of the residual variability after subtraction of the template. $\boldsymbol{B}$, Example of comparison between the waveforms corresponding to the original template (blue), the first principal component (PC \#1; red), and the time derivative of the template (green). $C$, Distribution of amplitudes after a fitting with a lower-amplitude threshold of 0.01 . The left peak corresponds to noise events and the right peak to real spikes. $D, E$, Two examples of the comparison between the voltage signal on several electrodes (blue) and the prediction obtained by summing the different templates with fitted amplitudes (red). Temporal window sizes: $\boldsymbol{D}, 20 \mathrm{~ms} ; \boldsymbol{E}, 100 \mathrm{~ms}$. Calibration bars: $D, 40$ SNR units; $E$, 50 SNR units.

tor, and patterned via photolithography and argon sputtering of the ITO (Fig. 1). They were coated with a $1 \mu \mathrm{m}$ silicon nitride insulator layer and electroplated with platinum black to reach an impedance of $100-300 \mathrm{k} \Omega$ at $1-10 \mathrm{kHz}$. A piece of salamander retina was pressed against the array in a controlled manner to obtain extracellular recordings of the ganglion cell layer with $10 \mathrm{kHz}$ sampling on each channel (see Materials and Methods). Slow voltage fluctuations were removed from each channel, and the signal was normalized by the covariance matrix of the background noise on that channel (see Materials and Methods).

\section{Spike and template extraction}

We began by extracting a set of candidate spikes from the raw data and clustering them into groups putatively representing individual cells. The candidate spikes were detected by flagging all local minima on any of the 252 channels that exceeded a threshold of six times the SD of the spatially whitened noise (see Materials and Methods). This threshold was permissive enough to capture all potential spikes but also included noise fluctuations. Candidate spike times $\left\{t_{i}\right\}$ were taken from all local minima exceeding the threshold.

Our algorithm is based on an explicit representation of the raw waveform in terms of templates (described in the next section and in Materials and Methods). Templates were identified by a preliminary clustering focusing on the peak of the waveform across all electrodes (see Materials and Methods). The final cluster assignment of individual spikes was made later, after template fitting (see next section and Materials and Methods). Note that this is different from many other ap- proaches to spike sorting, in which the clustering itself is used to assign the spikes to different cells.

To cluster the spikes, we represented each flagged spike event by the vector of voltage values on all 252 electrodes at that time. These 252-dimensional vectors were divided into groups according to which electrode had the largest voltage value for a given spike. Within each group, we then clustered the events using a modified mean-shift clustering algorithm, in which each point is assigned to a local maximum of probability density by gradient ascent (see Materials and Methods).

This clustering gives a rough sense of which spikes belong to which cells, but it typically does not assign the correct cluster when spikes occur synchronously. This is because the combined voltage waveform of synchronous spikes overlaps and does not resemble the waveform of any single spike. To attack the problem of overlapping spikes, we found the archetypal "template" voltage waveform on the array $\mathbf{w}_{j}(t)$ for each cluster $j$ and then fit all the putative spike events in the raw data to a combination of such templates (Eq. 3). We defined the template for a cluster $j$ as the point-wise median of all the spike waveforms in the cluster, in which the spike waveform for an event time $t_{i}$ consists of a $6.5 \mathrm{~ms}$ sample on all channels centered on $t_{i}$ (examples in Fig. $2 F, G$ ). Notice that each template has $252 \times 65$ components.

Note that the large number of electrodes preclude any attempt of doing the entire clustering by hand, as it has been done in several methods (Segev et al., 2004; Prentice et al., 2011).

\section{Fitting the templates}

We next use the templates to fit the raw data. Our algorithm is based on a model in which the extracellular recording is given 
A

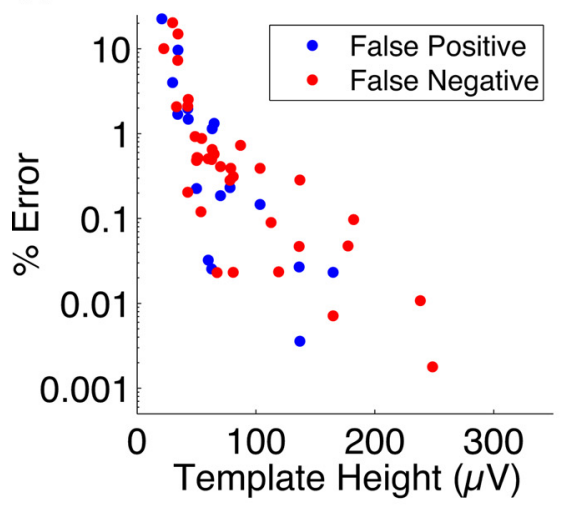

C

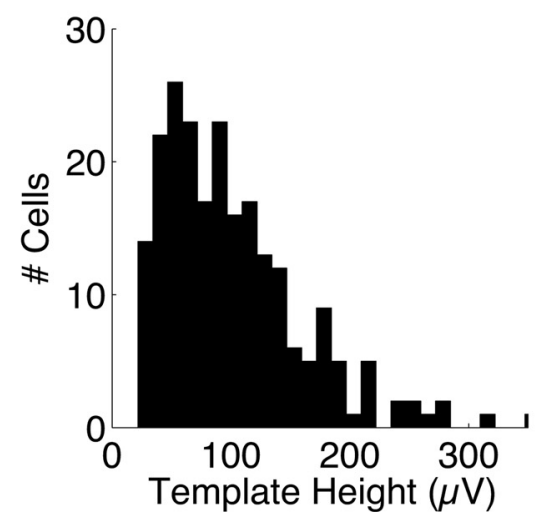

B

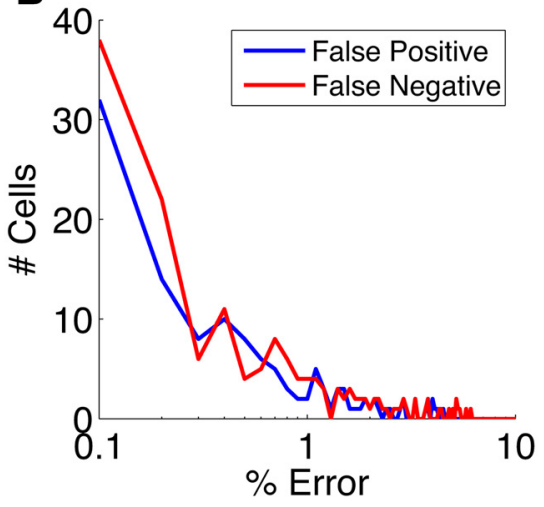

D

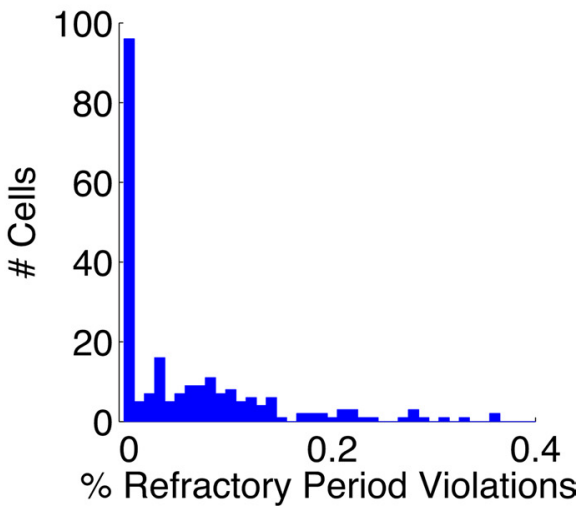

Figure 4. A, Frequency of sorting errors during the surrogate tests, as a function of the amplitude of the template for falsepositive (blue) and false-negative (red) errors. $\boldsymbol{B}$, Distribution of the frequency of false-positive (blue) and false-negative (red) errors deduced from the template amplitude distribution $(\boldsymbol{C})$ and the error rates as a function of amplitude $(\boldsymbol{A})$. $\boldsymbol{C}$, Distribution of the template amplitudes for one experiment. $\boldsymbol{D}$, Distribution of the fraction of interspike intervals that violate the refractory period for the same experiment.

by a linear superposition of neuronal activity from different cells:

$$
\mathbf{s}(t)=\sum_{i j} a_{i j} \mathbf{w}_{j}\left(t-t_{i}\right)+\mathbf{e}(\mathrm{t})
$$

where $s(t)$ is the signal recorded over 252 electrodes and over multiple time points (see below), $\mathbf{w}_{j}\left(t-t_{i}\right)$ is the template defined previously, $t_{i}$ are all the putative spike times over all the electrodes, $a_{i j}$ are amplitude factors that vary for each example of a spike time $t_{i}$ for cluster $j$, and $\mathbf{e}(t)$ is the background noise, which is not Gaussian (see Materials and Methods; Fee et al., 1996). Note that, although a previous attempt (Segev et al., 2004) assumed that the raw voltage traces could be modeled by a linear sum of templates with fixed amplitudes, here we allowed the amplitudes to be variable, reflecting the fact that depolarization in rapidly spiking neurons can lead to decrement in spike amplitude.

An important assumption of this model is that spike waveforms exhibit a multiplicative noise, captured by the amplitude factors $a_{i j}$, but have no other variation in the temporal shape of their waveform. To test this assumption, we selected spikes that were isolated (after spike sorting was completed), so that voltage variations did not arise from spikes emitted by other nearby cells. For these subsets, we found that the template model accounted for $92 \pm 4 \%$ (SD, $n=78$ cells) of the explainable voltage variability (see Materials and Methods, Quantifying goodness of fit). After subtracting the scaled template, we performed principal components analysis on the residuals. The first principal component accounted for $2 \%$ of the voltage variability (averaged across cells), showing that the residual shape variability could not be described in a low-dimension space (Fig. $3 A$ ). Furthermore, this first principal component was identical in shape to the time derivative of the template itself (Fig. $2 B)$, which is a typical effect arising from discrete sampling of a continuous voltage process (Fee et al., 1996). So in our recordings, the shape of the spike waveform had negligible variation, and the form assumed in Equation 3 was an excellent approximation.

At this point, all the parameters except the $a_{i j}$ have been determined by clustering and spike detection. Most of the $a_{i j}$ should be zero, because not all $t_{i}$ represent actual spikes and because, at any point in time, we expect that only a few of all the possible cells to spike synchronously. Conversely, nonzero values of $a_{i j}$ should all be close to 1 , because they indicate the presence of single spike from template $j$ with an amplitude close to the average value for that cell. Unfortunately, standard least-squared optimization methods do not take advantage of this previous expectation and would often produce fits involving many templates with very small amplitudes.

We therefore used an iterative greedy approach to estimate the $a_{i j}$ for each subgroup $t_{i}$, which bears some similarity to the matching pursuit algorithm (Mallat and Zhang, 1993). The $a_{i j}$ were determined by fitting the waveform; because of temporal overlap among spikes, the optimal values for the $i$ th spike may be affected by the fit of the $i+$ first spike, and so this is best treated as a large-scale simultaneous optimization problem. The fitting was performed in blocks of putative spike times, $\left\{t_{i}\right\}$, where each block consisted of a set of spikes with interspike time intervals $<6.5 \mathrm{~ms}$, because such spikes might suffer temporal overlap. To define these blocks, we labeled all the intervals between consecutive spike times $t_{i}$ that were larger than $6.5 \mathrm{~ms}$. Then we just grouped together in one block all the spike times between two labeled intervals.

Each block was then processed according to the following steps. (1) Estimate the normalized scalar products $\mathbf{s}(t) \cdot \hat{\mathbf{w}}_{j}\left(t-t_{i}\right)$ for each template $j$ and putative spike time $t_{i}$, for all the $i$ and $j$ in the block of raw data. (2) Choose the $(i, j)$ pair with the highest scalar product, excluding the pairs $(i, j)$ that have already been tried and the $t_{i}$ already explored (see below). (3) Set $a_{i j}$ equal to the amplitude value that best fits the raw data: $a_{i j}=\frac{\mathbf{s}(t) \cdot \hat{\mathbf{w}}_{j}\left(t-t_{i}\right)}{\left|\mathbf{w}_{j}\left(t-t_{i}\right)\right|}$. (4) Check whether the $a_{i j}$ amplitude value is between $a_{j}^{\min }$ and $a_{j}^{\max }$. (5) If so, then accept this value, subtract the scaled template from the raw data, $\mathbf{s}(t) \rightarrow \mathbf{s}(t)-$ $a_{i j} \mathbf{w}_{j}\left(t-t_{i}\right)$, and return to step 1 to re-estimate the scalar products on the residual. (6) Otherwise, increase by one $n_{i}$, which counts the number of times any template has been rejected for spike time $t_{i}$. If $n_{i}$ reaches $K=3$, label this $t_{i}$ as "explored." If all $t_{i}$ have been explored, quit the loop. Otherwise, return to step 1 and iterate. 
The reason to keep track of $n_{i}$ and have $K>1$ is the following. Sometimes two different templates could have similar shape but very different amplitudes. As a consequence, during the fitting process, the large template could have been chosen to fit a small event and rejected because of mismatch in amplitudes. If $K=1$, the corresponding time $t_{i}$ would be labeled as explored, and the algorithm would not have a chance to try the small template, which would be the right one in this example. Having $K>1$ enables the small template to be fitted properly. $K=3$ was chosen because, in our experience, this is the maximum number of nearby templates $k$ that have very similar shape $\left(C_{j k}=\hat{\mathbf{w}}_{j}(t)\right.$. $\hat{\mathbf{w}}_{k}(t) \geq 0.9$; see below for justification) to any given template $j$.

This algorithm has similarities to the projection pursuit algorithm (Mallat and Zhang, 1993) and to an iterative thresholding algorithm (Blumensath and Davies, 2008) in which we would try to fit the data while minimizing the $L_{0}$ norm of the amplitudes, i.e., the number of firing spikes. Note that the criterion used previously (Segev et al., 2004) was also related to a similar minimization. In comparison, a more classical $L_{1}$ minimization would produce a lot of nonzero $a_{i j}$ but with very small values. This would not fit our requirement that $a_{i j}$ coefficients are either 0 or close to 1 .

The free parameters of the algorithm are the amplitude thresholds $a_{j}^{\min }$ and $a_{j}^{\max }$. The upper threshold was set to 6 (in noise-normalized units), because none of the spikes we recorded have ever exceeded this amplitude. Selecting the lower threshold was more difficult, because it must be high enough to reject noise but not so high that it rejects real spikes. To find the right balance, we first fit the data with a "test" lower bound of 0.01 for all the templates. This threshold was always too low, but it produced a bimodal amplitude distribution (Fig. 3C) from which a "noise" peak and a "real spike" peak can be distinguished. We then set automatically the $a_{j}^{\min }$ for each template as the smallest local minimum in this amplitude distribution and then performed the entire fitting procedure again.

After fitting, we used a second run of the clustering algorithm to separate out additional templates, followed by hand adjustments to split some templates, as needed (see Materials and Methods). After rerunning the algorithm with an expanded set of templates, we merged clusters coming from the same ganglion cell following previous criteria (see Materials and Methods).

The end result of this fitting process was a very close match to the raw data (Fig. $3 C, D$ ). In particular, this good agreement validates the assumption of the model that the voltage waveforms from different spikes sum linearly. For all segments of raw data that had putative spikes, the fraction of variance explained by our fit (Eq. 2) was very high $\left(R^{2}=0.97 \pm 0.01, n=10,000\right.$ segments).

\section{Fitting performance and correlation estimation}

To evaluate the accuracy of our algorithm, we first used a method common in the literature to measure the overlap of spike waveform clusters (Pouzat et al., 2002; Rinberg et al., 2003; Hill et al., 2011). We fitted each cluster to a multivariate Gaussian distribution and then estimated the probability that events of template $i$ could be attributed to template $j$ for each $i$ and $j$ (see Materials and Methods). This probability was typically very low. For $90 \%$ of the templates, all the mixing probabilities with all other neighbors were below $5 \%$, indicating that our templates were well separated. However, these values should not be taken as absolute estimates of the error, because our algorithm does not rely on the noise covariance matrix to assign spike times to cells.

We estimated the fraction of spikes that would be missed because they would be lower than the threshold used for spike de-
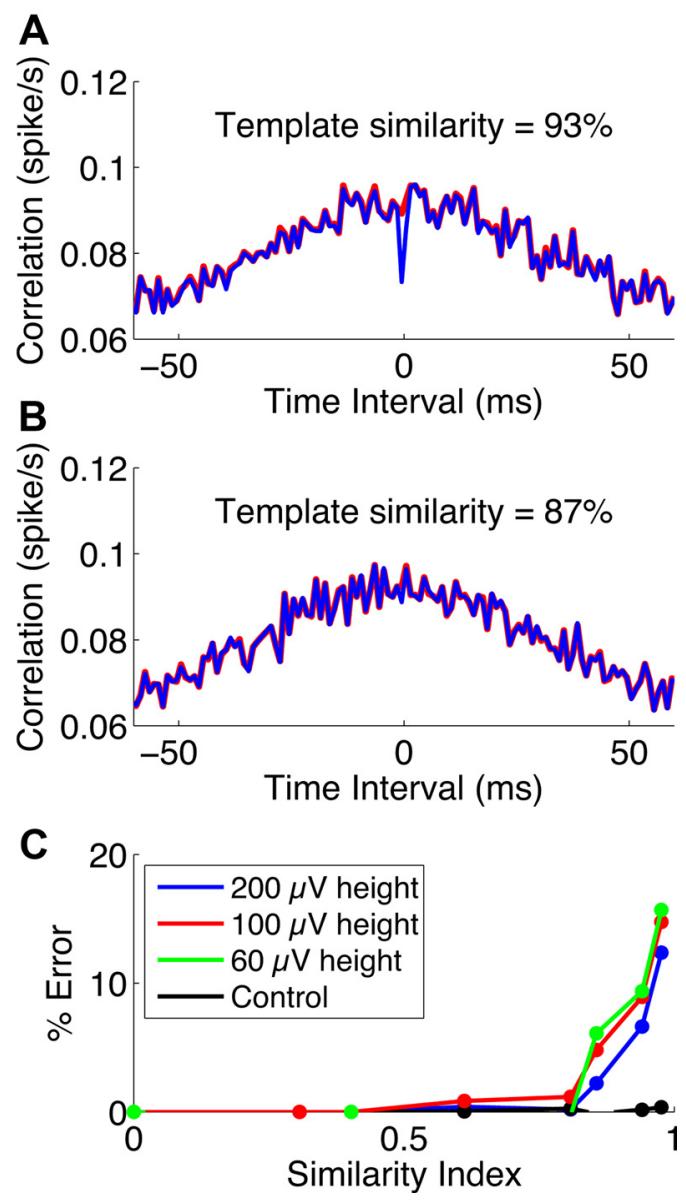

Figure 5. $A, B$, Example of the cross-correlogram between two surrogate spike trains for two pairs of templates having similarities of 0.93 and 0.87 , respectively. Red, The simulated spike train. Blue, The spike train recovered by the spike sorting. C, Relative error when estimating the cross-correlogram for time intervals between 2 and $5 \mathrm{~ms}$ (black) or time intervals between - 1 and $1 \mathrm{~ms}$ (green, red, and blue, with the three colors corresponding, respectively, to templates with amplitudes of 60,100 , and $200 \mu \mathrm{V})$.

tection. For this calculation, we assumed that the amplitude distribution of each cell was a Gaussian. Computing the mean amplitude of the spike and its SD, we estimated the probability of having a spike below the $6 \sigma$ threshold. This probability was below $0.1 \%$ for $91 \%$ of the cells.

A better test of the accuracy of our algorithm can be made by adding artificial templates, with variable amplitude, at known times to the raw data and rerunning our spike-sorting algorithm. We generated these artificial templates by spatially displacing a real template over the electrode array and chose its amplitude variation uniformly distributed with an SD (0.12) typical of that found in our experimental recordings $(0.176 \pm 0.077)$. We then ran the fitting algorithm on this modified data and counted how often it missed the added template (false negative) or detected it spuriously (false positive). As shown in Figure $4 A$, the falsepositive and false-negative rates were $<0.2 \%$ when the peak amplitude of the template was $>100 \mu \mathrm{V}$. Error rates were $<2.5 \%$ when peak amplitudes were $>35 \mu \mathrm{V}$. Almost all the templates (93\%) extracted from our recordings had peak amplitudes $>35$ $\mu \mathrm{V}$ (Fig. $4 B, C$ ), indicating that our algorithm could identify spikes with very high accuracy even when superimposed on data containing many other spikes. In addition, we counted the number of refractory period violations in sorted spike trains, with violations defined as spike intervals $<2 \mathrm{~ms}$. Half of the cells 


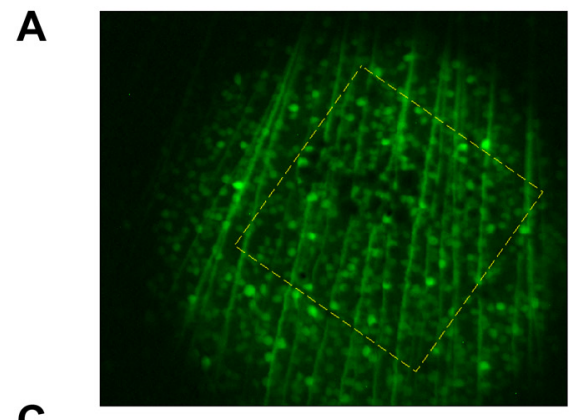

C
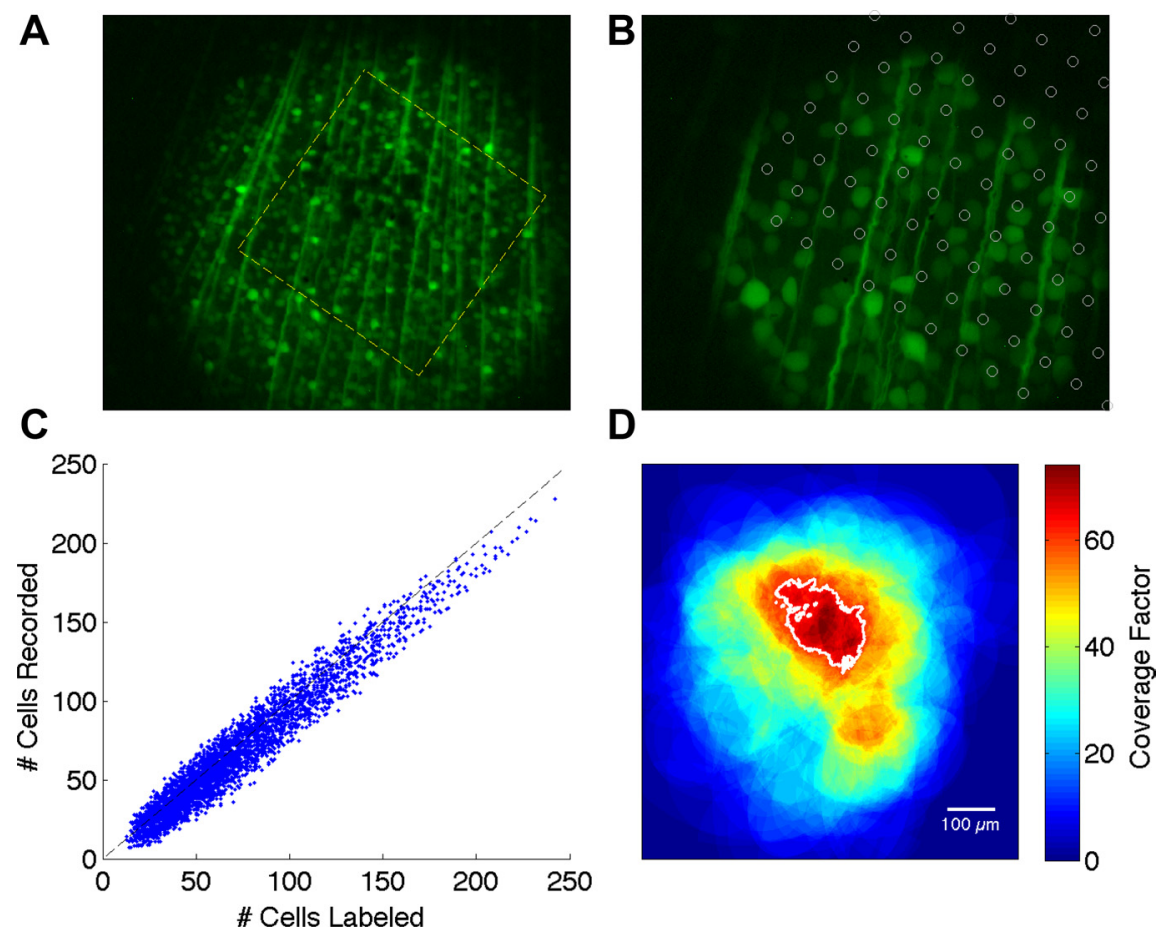

Figure 6. A, Example of a retinal patch over the multielectrode array. The ganglion cells are stained with rhodamine dextran, shown here in green. The dashed yellow line shows the borders of the array. $\boldsymbol{B}$, Close-up of the same retina, focused on the ganglion cell layer. The circles represent the position of the electrodes, imaged in a different focal plane. $\boldsymbol{C}$, Number of ganglion cells found from spike sorting plotted against the number of cells counted with the labeling. Each point represents a different subregion of the array. $\boldsymbol{D}$, Coverage map of visual space. The color indicates the coverage factor, defined as the number of receptive fields (at $1-\sigma$ radius) that overlap with a given pixel. White contour indicates region of complete coverage.

showed no refractory period violations, and $92 \%$ of the cells showed $<0.2 \%$ of them (Fig. $4 D$ ).

These robustly low error rates show that the fitting part of our algorithm correctly identifies most spikes (including overlapping ones), but they do not rule out the possibility that specific pairs of cells may be poorly detected when they spike together, leading to biases in some cell-cell correlations. To address this issue, we added pairs of artificial templates to the raw data at random but correlated times. To create artificial templates with high overlap, the second template was a version of the first displaced by less than one electrode by interpolation. When adding pairs of synthetic templates to the data, we needed a way to continuously vary their similarity. For this, we started with a first synthetic template $\mathbf{w}_{0}(x, y, t)$, where $x$ and $y$ are horizontal and vertical electrode coordinates and $t$ is time in the spike waveform, and generated a second template by displacing the first template by a distance of less than one electrode through interpolation. That is, we defined $\mathbf{w}_{\delta}(x, y, t)=(1-\delta) \mathbf{w}_{0}(x, y, t)+\delta \mathbf{w}_{0}(x+1, y+1, t)$, where $\delta<1$ and locations that were off of the array, like $\mathbf{w}_{0}\left(x_{\max }+1, y, t\right)$ were defined as 0 .

We then reran the spike-sorting algorithm on this surrogate data, reconstructed the cross-correlogram between these templates, and compared it with the real cross-correlogram (Fig. $5 A, B)$. We found that the correlation was well estimated when the template similarity (normalized cross-correlation $C_{i j}$ ) was $<0.8$. Above this value, there was an increasing artifactual dip in the cross-correlogram for a delay of $<1 \mathrm{~ms}$ that becomes visible for similarity $>0.9$ (Fig. $5 B$ ). The rest of the cross-correlogram was correctly estimated. The relative size of this dip increased with the similarity between the two templates but depended only marginally on the template amplitude (Fig. 5C).
What was the impact of this misestimation on our data? For pairs of cells with template similarity $>0.8$, the value of the cross-correlogram for a delay between -1 and +1 ms could be underestimated. For each pair of cells that could be problematic ( similarity of the templates $>0.8$ ), we did a linear interpolation to estimate the true value of the cross-correlation for a delay between -1 and +1 ms. We found a slight difference between the interpolated and the measured values, corresponding on average to $0.2 \%$ of the spikes. The impact of our underestimation bias was thus minimal.

In conclusion, realistic tests of our algorithm show that it successfully solves the problem of identifying overlapping spikes making very few sorting errors and gives minimally biased estimates of correlation for all but a very few cases.

\section{Completeness of our recordings}

To measure what fraction of cells we recorded from, we took advantage of the transparency of our arrays to combine the extracellular recording with epifluorescence imaging of the same patch of retina (Fig. 6A,B). We loaded the ganglion cells selectively with rhodamine dextran through the optic nerve (see Materials and Methods), imaged them with epifluorescence after the recording, and counted the number of cells over the array. Our cell count implied an average density of $1320 \pm 90$ cells $/ \mathrm{mm}^{2}$ ( $n=4$ retinas), in agreement with previous estimates (Segev et al., 2004; Behrend et al., 2009), indicating that our results are consistent with loading all the ganglion cells with the dye.

We used the algorithm described above to sort spikes from the recording and counted the number of valid templates, each of which corresponds to a single recorded ganglion cell. In one recording, excluding the borders of the arrays, we found a total of 228 valid templates compared with 242 cells in the epifluorescence labeling, suggesting that we recorded $94 \%$ of the cells in our patch of retina. To provide additional evidence that the templates produced by spike sorting correspond to real ganglion cells, we compared the spatial distribution of the templates with that of the labeled cells. We considered subregions of the array consisting of rectangular areas containing different numbers of electrodes (minimum $n=9$ ). For each subregion, we counted the number of sorted cells whose templates had their peak voltage on one of the electrodes in that subregion. Then, we compared against the number of labeled cells within that subregion, including a border area spanning halfway to the next electrodes. The results, shown in Figure 6C, demonstrate a close match between the number of cells sorted and cells labeled, even over the smallest subregions. These results strongly suggest that we recorded almost all the cells above the array. Another recording gave qualitatively similar results with $\sim 80 \%$ of the templates recorded.

\section{Coverage map}

These results show that we were able to record virtually all the cells above the array. We next sought to measure the coverage factor associated with this set of cells. We estimated the receptive field of 
each cell from its response to a randomly flickering checkerboard, fitted a twodimensional Gaussian curve to each of them, and defined their area from the $1-\sigma$ radius of this curve fit (Segev et al., 2004, 2006). The average coverage factor is the number of receptive fields that cover each pixel of visual space. We estimated this quantity by multiplying the average area of ganglion cell receptive fields $(46,200 \pm 1880$ $\mu \mathrm{m}^{2}, n=274$ cells) by the measured cell density for this experiment (1370 cells/ $\mathrm{mm}^{2}$ ). We obtained an average coverage factor of $63 \pm 2$. This coverage is consistent with a previously reported value (Segev et al., 2004).

Using the Gaussian fits, we then computed how many receptive fields covered each pixel of visual space and compared it with the previous estimate (Fig. $6 D$ ). We found a region (area $\sim 150 \times 200 \mu \mathrm{m}$; Fig. $6 \mathrm{D}$, white contour) with at least $90 \%$ of the expected receptive field coverage. We note that we could not measure the receptive field for $\sim 8 \%$ of the ganglion cells (see below), which reduced our measured coverage accordingly. So our methods allowed us, for the first time, to witness the activity of a highly complete population of ganglion cells sampling a region of the visual space. No comparably complete recording of neural activity at the resolution of single spikes of comparable completeness has been possible to date.
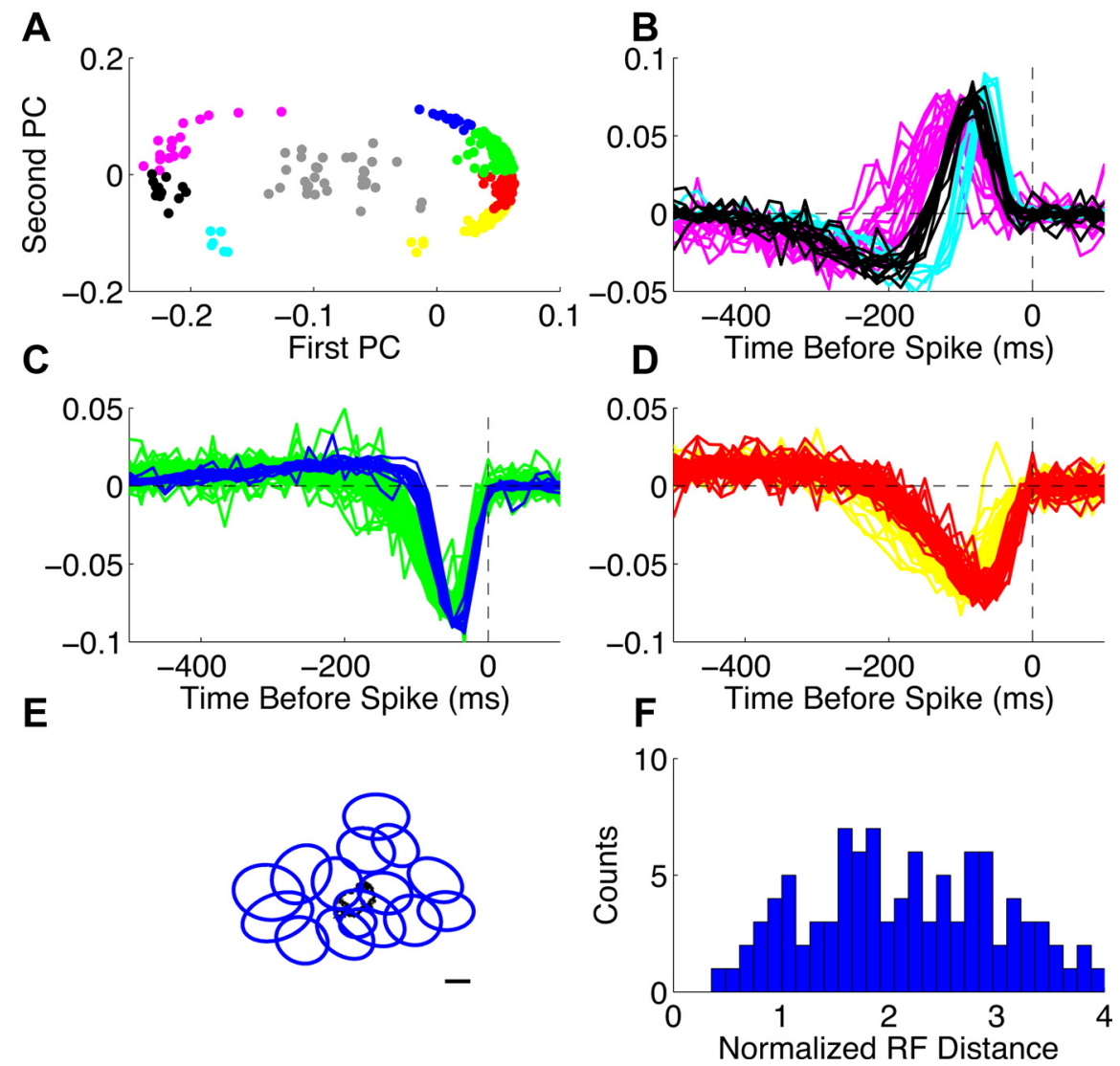

Figure 7. A, Projections of the receptive field temporal profiles on the first and second principal components (PC). Each dot represents one ganglion cell, colors indicate cell type, and gray indicates unclassified cells. $\boldsymbol{B}-\boldsymbol{D}$, Temporal profiles of the different cell types. B, Fast ON (cyan), medium ON (black), and slow ON (magenta). C, Biphasic OFF (blue) and monophasic fast OFF (green). $\boldsymbol{D}$, Medium OFF (red) and slow OFF (yellow). E, Ellipses fitted to the spatial receptive field profile for the biphasic OFF cells. Scale bar, $100 \mu \mathrm{m}$. $\boldsymbol{F}$, Distribution of the normalized receptive field distances between biphasic OFF cells.

\section{Cell types, tiling, and coverage}

This complete population can be divided into different functional types, using the temporal shape extracted from the full spatiotemporal receptive field for each cell (Devries and Baylor, 1997; Schnitzer and Meister, 2003; Segev et al., 2006) and $k$-means clustering (Segev et al., 2006). We confirmed the definition of clusters by doing a principal component analysis over all the temporal profiles and visualizing the projections onto the first two principal components (Fig. 7A). In this space, clusters were well separated, with a small fraction of cells having properties on the boundary between two clusters. Temporal profiles of ganglion cells from the same cluster were very similar to each other and generally separated from cells of different functional type (Fig. $7 B-D$ ). The existence of a small fraction of cells with ambiguous classification is an inherent property of this neural population, as reported previously (Segev et al., 2006).

One of the types, the fastest OFF (referred to as "biphasic OFF" by Segev et al., 2006), clearly tiled visual space, as shown by plotting the ellipses fitted for all the cells of this type (Fig. 7E). To quantify the tiling, we computed the distance between the receptive field centers for all cell pairs of the same type and normalized by the sum of their respective receptive field radii (Devries and Baylor, 1997; Segev et al., 2006). The distribution of these normalized distances showed a clear gap less than a normalized distance of 1 for this cell type (Fig. $7 F$ ). Nothing similar was observed for any other cell type (data not shown).

The completeness of our recordings allowed us to measure precisely the coverage factor for each cell type. They all had a coverage factor $>1: 3.2$ for the fast $\mathrm{ON}, 1.3$ for the medium $\mathrm{ON}$, 4.1 for the slow ON, 19.6 for the monophasic fast OFF, 11.7 for the medium OFF, and 20.1 for the slow OFF. The four types of OFF cells found in this study agree with previous reports; however, we observed one more type of ON cell than described previously (Segev et al., 2006). Although the medium ON cell had a coverage factor close to one, we still observed clear violations of tiling. Of course, we only divided cells into functional types using the temporal profile of their receptive field center. Additional divisions may be possible using other functional measures, although simple properties, such as receptive field center size, autocorrelation function, and response to steps of light, have been shown to confuse functional boundaries rather than delineate additional cell types in the salamander (Segev et al., 2006).

\section{Cells with weak receptive fields}

Our clustering of cells according to the temporal profile of their receptive fields left aside a significant portion of cells as unclassified $(8 \%)$. For these cells, the receptive field was typically dominated by noise in both space and time, so that the temporal profile in the spatial location with the extremal spike-triggered stimulus average had no discernible structure (Fig. $8 D$ ). We refer to this phenomenon as cells having "weak receptive fields." One possible explanation for measuring a weak receptive field is that the cell fired very few spikes during the checkerboard flicker ensemble. This was not always the case, because $50 \%$ of the weak receptive field cells had a firing rate higher than the average 


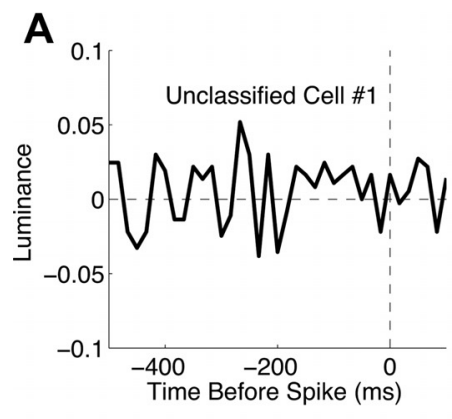

E

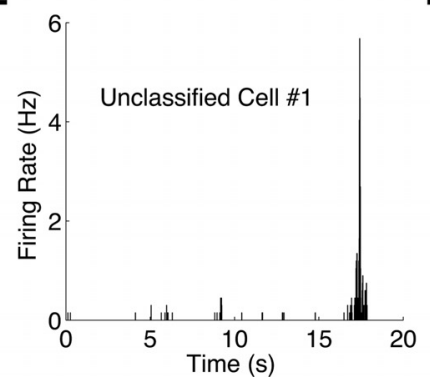

B

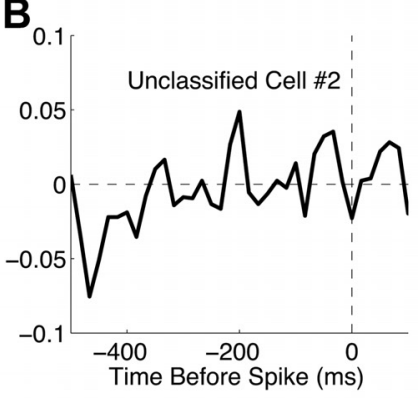

$\mathbf{F}$

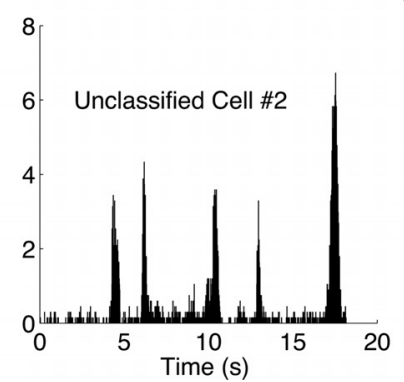

$\mathbf{C}_{0.1}$

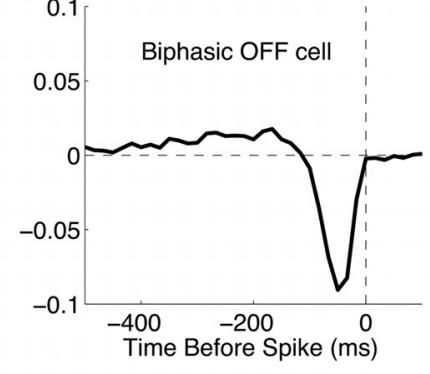

G

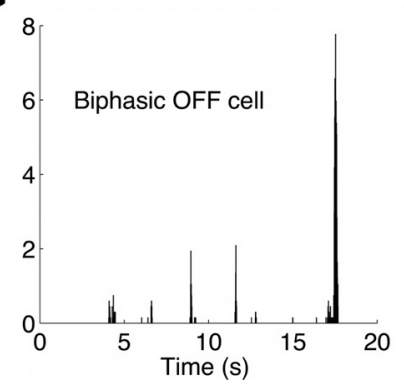

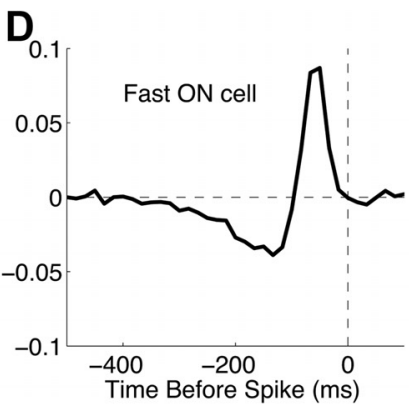

H

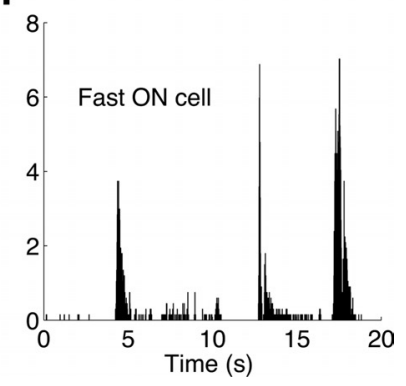

Figure 8. $\quad \boldsymbol{A}-\boldsymbol{D}$, Temporal receptive field profiles of four different ganglion cells. $\boldsymbol{E}-\boldsymbol{H}$, Firing rate (PSTH) of the same cells in response to a natural movie.

firing rate across the rest of the population during flicker $(0.8$ spikes/s) (Fig. 9).

Another possible explanation is that these cells might not respond reliably to visual stimulation [or not respond at all to transient changes, such as melanopsin-containing ganglion cells (Berson, 2003)]. In the same recording session, we displayed repeated presentations of a short (20 s) natural movie clip. The peristimulus time histograms (PSTHs) of these cells showed strong locking to events in the natural movie, comparable with cells with well-defined receptive fields. We also estimated the mutual information between the stimulus and spike times for each cell using a standard method (Brenner et al., 2000) already used in the retina (Schneidman et al., 2011) that computes $I=\frac{1}{T} \int_{0}^{T} \frac{r(t)}{\bar{r}} \log _{2} \frac{r(t)}{\bar{r}}$, where $r(t)$ is the firing rate as a function of time estimated from the PSTH, and $r$ is the mean firing rate. We found a wide variety of information rates, which tended to be anticorrelated with the average firing rate measured during the natural movie (Fig. 10). This measure showed no obvious difference between the cells with weak receptive fields and other cells. By showing that these cells responded as vigorously as others during naturalistic stimulation, we ruled out the possibility that they are not visually responsive or are non-imageforming light detectors, such as melanopsin-containing ganglion cells (Berson, 2003).

One possibility is that they are cells with a low SNR. This would be the case if they have a high spontaneous firing rate or if they show a broad tuning and a low gain. In both cases, these cells could have a wide range of overall firing rates, but their spikes would not be very informative about the stimulus.

We tested these hypotheses by repeating many times a single sequence of $30 \mathrm{~s}$ of the flickering checkerboard stimulus. The sequence was interleaved with non-repeated sequences of the checkerboard from which the receptive fields were measured. There was no difference between the firing rate of each cell during repeated and non-repeated sequences (Pearson's coefficient $r=$ 0.99). The PSTHs of all the cells showed reliable responses to the repeated sequence (Fig. 9). We estimated the information be- tween this stimulus and the spikes of each cell and found a behavior similar to that found for the natural movie stimulus (Fig. 10); the visual information encoded per spike (red dots) was anticorrelated with the mean firing rate and comparable with the values obtained for cells that had a clear receptive field (blue dots). Although these weak receptive field cells tended to have a slightly lower firing rate than the other ganglion cells, the visual information conveyed by these cells was comparable when matched for overall firing rate. It is interesting to note that the similarity of the values for weak receptive field cells, and the other ones, was higher for the natural movie stimulus than for the checkerboard stimulus.

For the cells with the lowest firing rates, an alternative explanation is that they are highly selective to some stimulus features that occur rarely during checkerboard flicker. To understand better the selectivity of these cells, we also recorded their responses to moving bars and gratings. All of the weak receptive field cells responded reliably to both of these moving stimuli, and their peak firing rates were comparable in magnitude with the other cells (mean peak firing rate for the bar, $27 \pm 3 \mathrm{~Hz}$; for the grating, $9 \pm 0.5 \mathrm{~Hz}$ ). In addition, the spatial and temporal frequency tuning of the weak receptive field cells were similar to the other ones (data not shown).

Another possibility is that the weak receptive field cells could encode a nonlinear function of the stimulus that embodies a symmetry between responses to $\mathrm{ON}$ and OFF events. In this case, equally likely responses to $\mathrm{ON}$ and OFF events would roughly cancel each other in the spike-triggered stimulus average. This hypothesis could not be directly tested, but we also recorded the response of these cells to spatially uniform ON and OFF flashes. For each cell, we estimated an ON/OFF index by dividing the peak $\mathrm{ON}$ response by the sum of the $\mathrm{ON}$ and OFF peak responses. Zero corresponds to a pure OFF response, and one corresponds to a pure $\mathrm{ON}$ response to the flash. Although individual ganglion cells exhibit a diversity of responses to these simple stimuli, the distribution of this index was similar for weak receptive field cells and for standard receptive field ones (Fig. 11A). In particular, many of the weak receptive field cells were not ON/OFF type in 

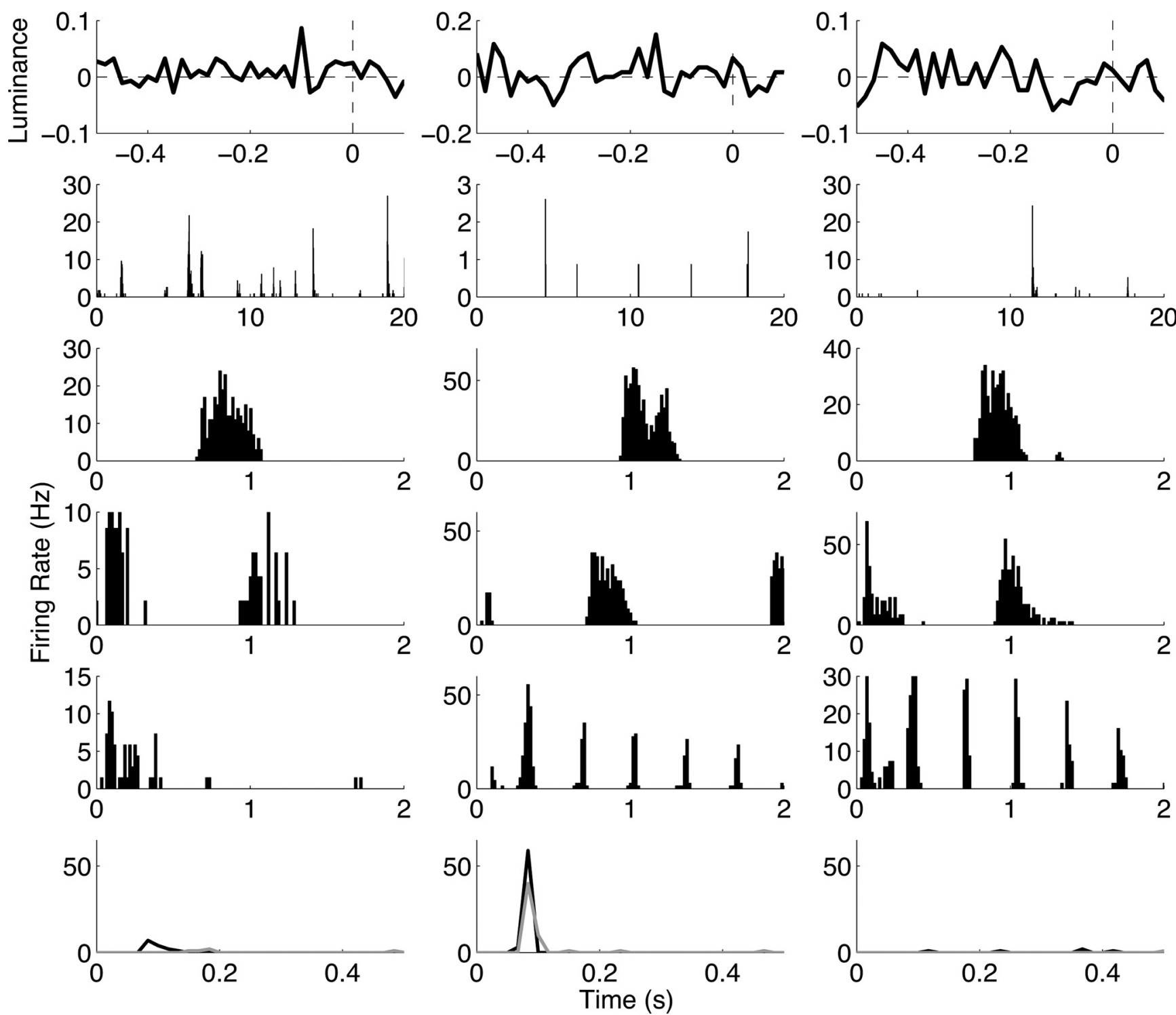

Figure 9. The responses of three different weak receptive field cells to a set of standard visual stimuli. Each column corresponds to one cell. First line, Temporal profile of the spike-triggered average. Second line, PSTH of the response to the repeated sequence of checkerboard flicker. Third line, PSTH of the response to the moving bar. Fourth and fifth lines, Response to two different gratings, with speeds of 2.6 and $3.64 \mathrm{~mm} / \mathrm{s}$, respectively, and spatial frequency of 1.66 cycles/mm. Note that the grating appears a time 0 and then immediately starts to drift, hence the transients that are sometimes seen. Sixth line, PSTH of the response to light (gray) and dark (black) spatially uniform flashes.

their responses to uniform steps of light, indicating that there was not necessarily any symmetry between wide-field ON and OFF stimulus events that caused spikes.

A final possibility is that weak receptive field cells might have unusual properties of their surround, including stronger suppression than for other ganglion cells. We next estimated a flash sensitivity index, defined as the ratio between the peak rate in response to flashes (maximum of the ON and OFF responses) and the peak response to the moving bar. For most cells, this index was below one (Fig. $11 \mathrm{~B}$ ), but the distribution was similar between standard cells and weak receptive field cells, with no significant difference. Thus, weak receptive field cells did not seem to have unusually strong receptive field surrounds.

\section{Discussion}

We have shown that the combination of a large, dense multielectrode array and a novel spike-sorting algorithm allowed us to record simultaneously from $>90 \%$ of a highly overlapping pop- ulation of $200+$ retinal ganglion cells. Crucially, for such large datasets, most of the action of the algorithm was performed in an automated manner. Although human intervention was still required at the end, the number of steps scaled linearly with the number of cells, making it feasible for our large-scale recordings. Because of the extremely low rates of spike-sorting errors and the low distortion of cross-correlation functions between neurons, these data will allow an investigation of how collective states of the neural population encode visual information. In the salamander retina, a single region of visual space maps onto a population of ganglion cells covering $\sim 200 \times 200 \mu \mathrm{m}$ and numbering $\sim 200$ cells. This implies that our technique captured a neural population that forms an essentially complete representation of a region of the visual space, as shown by our coverage map analysis. Of course, ganglion cells off of the array can still be activated through their receptive field surround, so our methods did not strictly capture every relevant ganglion cell. In any case, simultaneous recording of a neural population at this level of completeness is, 

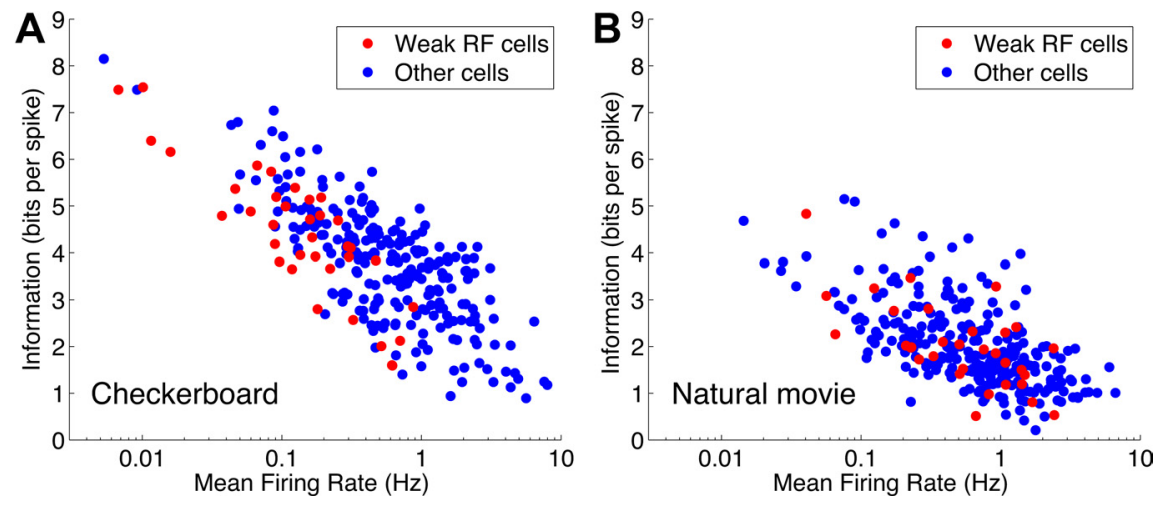

Figure 10. Mutual information between each cell and the stimulus, either checkerboard flicker $(\boldsymbol{A})$ or a natural movie clip (B), as a function of their mean firing rate. Red points, Weak RF cells. Blue points, All the other cells. RF, Receptive field.
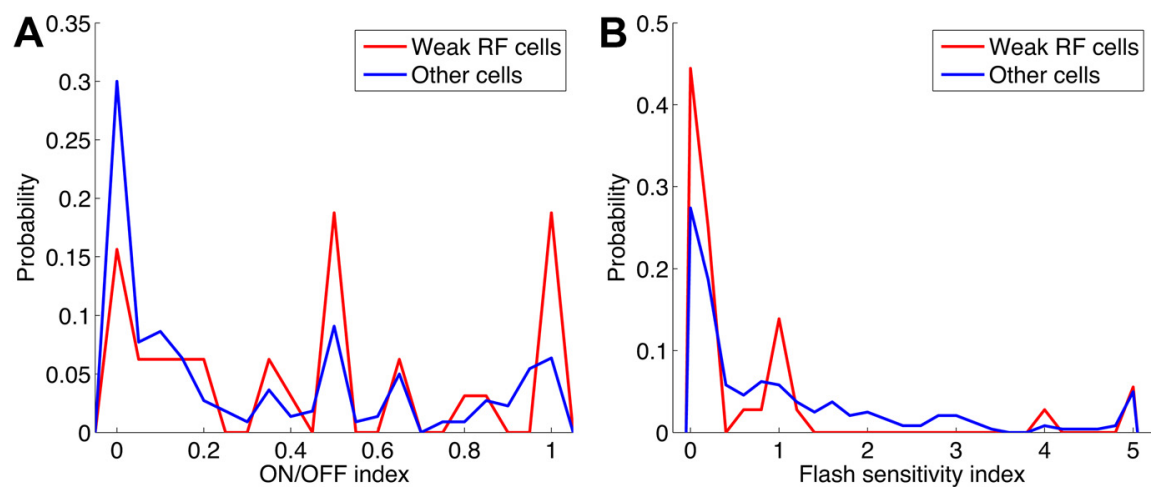

Figure 11. Histogram of the ON/OFF index ( $\boldsymbol{A}$ ) and of the flash sensitivity index (B) (see Results) for standard cells (blue) and weak receptive field (RF) cells (red).

to date, unprecedented in neuroscience. Our technique paves the way for the study of collective encoding of visual information by complete populations of retinal neurons, in which there is virtually no "hidden variables," i.e., unrecorded cells that would carry a significant part of the sensory information.

\section{Comparison with other spike-sorting methods}

The classical approach to extracellular spike sorting uses clustering of some aspect of the spike waveform to assign spikes to cells (Lewicki, 1998; Hill et al., 2011). The earliest methods used only spike amplitude (Hubel, 1957), with later methods adding spike width (Meister et al., 1994) and projections onto basis functions (Litke et al., 2004; Quiroga et al., 2004), followed by fitting the clusters with a mixture of Gaussians. These methods are typically quite successful when electrodes have been adjusted to isolate signals from one neuron, but larger-scale arrays of electrodes do not allow individual adjustment. Also, a single spike can be seen on many electrodes (Fig. 2F). This high dimensionality of the spike waveform makes a fit to a Gaussian mixture model quite challenging. More importantly, these datasets typically contain overlapping spikes from different neurons having comparable amplitude. Because the spike waveform shape is significantly changed by the occurrence of another spike at the same time, most of the overlapping spikes will be incorrectly sorted and the correlations between cells will be consequently biased. This is especially critical for dense recording arrays in which overlap is more often the rule than the exception.

Several algorithms have been proposed to handle overlapping spikes, but none of them has been fully tested at the large-scale level. In particular, they all used manual clustering algorithms that do not scale as readily to larger datasets. Most of them assume a fixed shape and amplitude for the template (Segev et al., 2004; Pillow et al., 2008), which increases the risk of errors. Prentice et al. (2011) recently used a likelihood test that assumes Gaussian statistics for the variable spikes amplitudes. Second, they used a goodness-of-fit criterion to decide whether a spike had been detected or not. The goodness-of-fit parameter was fixed to be the same for all cells, which is unlikely to capture the variety inherent in large datasets. Furthermore, the optimal value for this parameter can only be derived in the case of Gaussian noise (Prentice et al., 2011), and the background noise in extracellular recordings is far from Gaussian (Fee et al., 1996) because it is dominated by "neural hash," which reflects the activity of other neurons that are too far away to be recorded. It will be interesting to compare our algorithm with that of Prentice et al. (2011) in the future and possibly combine the best features of both approaches. In previous work, we showed a near-complete recording from an array of 30 electrodes (Segev et al., 2004). However, these arrays were too small to record from a neural population that completely covers a region of visual space.

The techniques described here can be readily applied to the mammalian retina. The prerequisite to record almost all the ganglion cells is that they should be organized in a single layer and that the density of the ganglion cells roughly matches the density of the electrodes. Most mammalian retinas have a one-cell-thick ganglion cell layer in the periphery, and the cell density will match the array at some eccentricity (guinea pig, Do-Nascimento et al., 1991; rabbit, Oyster et al., 1981; cat, Hughes, 1981; not the mouse, in which cell density is too high). In addition to the retina, our method can be applied to in vitro systems, such as the olfactory epithelium (Holy et al., 2000), hippocampal slices (Novak and Wheeler, 1988), and cell cultures (Hafizovic et al., 2007), in which smaller arrays have been used previously. Our sorting method is scalable, because the entire spike-sorting algorithm can be parallelized with high efficiency. So our methods can be applied to the next generation of recording devices with several thousands of electrodes (Imfeld et al., 2008), to get even bigger retinal areas sampled with full coverage. The algorithm could also be useful for in vivo applications, as long as the different electrodes are close enough so that one spike is seen on many electrodes.

\section{Weak receptive field cells}

We found a class of ganglion cells that we named weak receptive field cells, because we could not find a clear spike-triggered stimulus average during $\sim 1 \mathrm{~h}$ stimulation with checkerboard flicker, a common technique for mapping receptive fields (Meister et al., 1994; Devries and Baylor, 1997; Chichilnisky, 2001). We found that these cells represent $\sim 8 \%$ of the total ganglion cell population in the retina. This is, to our knowledge, the first comprehen- 
sive account of the prevalence of such cells in the retina. By showing that these cells responded vigorously during both repeated checkerboard flicker and naturalistic stimulation, we ruled out the possibility that these ganglion cells are not visually responsive or are non-image-forming light detectors, such as melanopsin-containing ganglion cells (Berson, 2003).

We raised the possibility that weak receptive field cells could be ON/OFF cells, in which responses during checkerboard flicker are roughly balanced between $\mathrm{ON}$ - and OFF-type stimulus events. Note that, although this behavior has been described for complex cells in the visual cortex (Rust et al., 2005), it has not been reported in the retina. However, our measurement of responses to spatially uniform steps of light revealed that many weak receptive field cells were not ON/OFF cells, thus ruling out this possibility as the sole explanation.

Another possibility is that these cells have highly nonlinear spatial subunits in their receptive fields, which presumably are individual bipolar cells (Demb et al., 2001). Bipolar cells are known to have center-surround spatial receptive fields themselves (Werblin and Dowling, 1969; Dacey et al., 2000; Baccus et al., 2008). If this center-surround antagonism is strong enough, then a check at one spatial location could cause the ganglion to fire either when it is bright or dark. For instance, a dark check on the center mechanism of an OFF-type bipolar cell could stimulate that bipolar cell directly and thus drive the ganglion cell to spike. However, a bright check on that same location could fall in the surround of another OFF-type bipolar cell and help disinhibit that cell strongly enough to cause a ganglion cell spike. In this circumstance, there could still be a rough symmetry between the number of ganglion cell spikes driven by this check taking a dark value versus taking a bright value, resulting in cancellation in the spike-triggered stimulus average. However, such a ganglion cell would still receive input only from OFF-type bipolar cells, giving it an OFF-type response to spatially uniform steps of light. A similar mechanism could result from ON bipolar cell input as well.

In several regions of sensory cortex, there have been reports of "nonclassical neurons" that were not responsive to classical stimuli, such that receptive field maps were not found. In the somatosensory cortex, more cells were found without classical receptive fields than with them (Dykes and Lamour, 1988). In the barrel cortex, a significant proportion of cells had a spiking probability in response to stimulation below or equal to their spontaneous firing rate (Kerr et al., 2007). In the adult mouse visual cortex, a recent report showed that $\sim 60 \%$ of the cells did not respond to visual stimulation with gratings, and $\sim 75 \%$ did not respond for strong luminance changes (Rochefort et al., 2011). Another report identified $13 \%$ of all neurons recorded extracellularly in mouse V1 did not respond to a battery of stimuli, including gratings, bars, and random flicker (Niell and Stryker, 2008). So far, it is still unknown what is the exact proportion of these cells in an unbiased sampling, nor is it known what properties they can encode.

Here we found $\sim 8 \%$ of such ganglion cells in the retina. Given the long history of retinal physiology, it is perhaps somewhat surprising that such a large fraction of ganglion cells with unknown function still exist. Two features of our technique were critical to reaching this conclusion: (1) the completeness of the recording and (2) the very low estimated rate of spike-sorting errors that allowed us to record cells with very low firing rate. Furthermore, we showed that these weak receptive field cells encode substantial visual information about both naturalistic and artificial stimuli, so they are likely to be important components of the population code of the retina. Future studies will need to address what stimulus features drive their firing as well as find better ways to identify them.

\section{References}

Averbeck BB, Latham PE, Pouget A (2006) Neural correlations, population coding and computation. Nat Rev Neurosci 7:358-366.

Baccus SA, Olveczky BP, Manu M, Meister M (2008) A retinal circuit that computes object motion. J Neurosci 28:6807-6817.

Barnes S, Werblin F (1986) Gated currents generate single spike activity in amacrine cells of the tiger salamander retina. Proc Natl Acad Sci U S A 83:1509-1512.

Behrend MR, Ahuja AK, Humayun MS, Weiland JD, Chow RH (2009) Selective labeling of retinal ganglion cells with calcium indicators by retrograde loading in vitro. J Neurosci Methods 179:166-172.

Berson DM (2003) Strange vision: ganglion cells as circadian photoreceptors. Trends Neurosci 26:314-320.

Blumensath T, Davies ME (2008) Iterative thresholding for sparse approximations. J Fourier Anal Appl 14:629-654.

Borkholder D (1998) Cell based biosensors using microelectrodes. $\mathrm{PhD}$ thesis, Stanford University.

Brenner N, Strong SP, Koberle R, Bialek W, de Ruyter van Steveninck RR (2000) Synergy in a neural code. Neural Comp 12:1531-1552.

Chichilnisky EJ (2001) A simple white noise analysis of neuronal light responses. Network 12:199-213.

Comaniciu D, Meer P (2002) Mean shift: a robust approach toward feature space analysis. IEEE Trans Pattern Anal Mach Intell 24:603-619.

Dacey D, Packer OS, Diller L, Brainard D, Peterson B, Lee B (2000) Center surround receptive field structure of cone bipolar cells in primate retina. Vision Res 40:1801-1811.

Demb JB, Zaghloul K, Haarsma L, Sterling P (2001) Bipolar cells contribute to nonlinear spatial summation in the brisk-transient $(\mathrm{Y})$ ganglion cell in mammalian retina. J Neurosci 21:7447-7454.

Devries SH, Baylor DA (1997) Mosaic arrangement of ganglion cell receptive fields in rabbit retina. J Neurophysiol 78:2048-2060.

Do-Nascimento JL, Do-Nascimento RS, Damasceno BA, Silveira LC (1991) The neurons of the retinal ganglion cell layer of the guinea pig: quantitative analysis of their distribution and size. Braz J Med Biol Res 24:199214.

Dykes R, Lamour Y (1988) Neurons without demonstrable receptive fields outnumber neurons having receptive fields in samples from the somatosensory cortex of anesthetized or paralyzed cats and rats. Brain Res 440: 133-143.

Fee MS, Mitra PP, Kleinfeld D (1996) Variability of extracellular spike waveforms of cortical neurons. J Neurophysiol 76:3823-3833.

Frechette ES, Sher A, Grivich MI, Petrusca D, Litke AM, Chichilnisky EJ (2005) Fidelity of the ensemble code for visual motion in primate retina. J Neurophysiol 94:119-135.

Fukunaga K, Hostetler LD (1975) The estimation of the gradient of a density function, with applications in pattern recognition. IEEE Trans Inf Theory 21:32-40.

Hafizovic S, Heer F, Ugniwenko T, Frey U, Blau A, Ziegler C, Hierlemann A (2007) A CMOS-based microelectrode array for interaction with neuronal cultures. J Neurosci Methods 164:93-106.

Hahnloser RH, Kozhevnikov AA, Fee MS (2002) An ultra-sparse code underlies the generation of neural sequences in a songbird. Nature 419:65-70.

Harris KD, Henze DA, Csicsvari J, Hirase H, Buzsáki G (2000) Accuracy of tetrode spike separation as determined by simultaneous intracellular and extracellular measurements. J Neurophysiol 84:401-414.

Hill DN, Mehta SB, Kleinfeld D (2011) Quality metrics to accompany spike sorting of extracellular signals. J Neurosci 31:8699-8705.

Holy TE, Dulac C, Meister M (2000) Responses of vomeronasal neurons to natural stimuli. Science 289:1569-1572.

Hubel DH (1957) Tungsten microelectrode for recording from single units. Science 125:549-550.

Hubel DH, Wiesel TN (1962) Receptive fields, binocular interaction and functional architecture in the cat's visual cortex. J Physiol 160:106-154.

Hughes A (1981) Population magnitudes and distribution of the major modal classes of cat retinal ganglion cell as estimated from HRP filling and a systematic survey of the soma diameter spectra for classical neurones. J Comp Neurol 197:303-339. 
Imfeld K, Neukom S, Maccione A, Bornat Y, Martinoia S, Farine P, KoudelkaHep M, Berdondini L (2008) Large-scale, high-resolution data acquisition system for extracellular recording of electrophysiological activity. IEEE Trans Biomed Eng 55:8.

Kerr JN, de Kock CP, Greenberg DS, Bruno RM, Sakmann B, Helmchen F (2007) Spatial organization of neuronal population responses in layer 2/3 of rat barrel cortex. J Neurosci 27:13316-13328.

Lewicki MS (1998) A review of methods for spike sorting: the detection and classification of neural action potentials. Network 9:R53-R78.

Litke AM, Bezayiff N, Chichilnisky EJ, Cunningham W, Dabrowski W, Grillo AA, Grivich M, Grybos P, Hottowy P, Kachiguine S, Kalmar RS, Mathieson K, Petrusca D, Rahman M, Sher A (2004) What does the eye tell the brain? Development of a system for the large scale recording of retinal output activity. IEEE Trans Nucl Sci 51:1434-1440.

Mallat SG, Zhang Z (1993) Matching pursuits with time-frequency dictionaries. IEEE Trans Signal Process 41:3397-3415.

Meister M, Pine J, Baylor DA (1994) Multi-neuronal signals from the retina: acquisition and analysis. J Neurosci Methods 51:95-106.

Miller RF, Staff NP, Velte TJ (2006) Form and function of on-off amacrine cells in the amphibian retina. J Neurophysiol 95:3171-3190.

Niell CM, Stryker MP (2008) Highly selective receptive fields in mouse visual cortex. J Neurosci 28:7520-7536.

Novak JL, Wheeler BC (1988) Multisite hippocampal slice recording and stimulation using a 32 element microelectrode array. J Neurosci Methods 23:149-159.

Ohki K, Chung S, Ch'ng YH, Kara P, Reid RC (2005) Functional imaging with cellular resolution reveals precise micro-architecture in visual cortex. Nature 433:597-603.

Oyster CW, Takahashi ES, Hurst DC (1981) Density, soma size, and regional distribution of rabbit retinal ganglion cells. J Neurosci 1:1331-1346.

Pillow JW, Shlens J, Paninski L, Sher A, Litke AM, Chichilnisky EJ, Simoncelli EP (2008) Spatio-temporal correlations and visual signalling in a complete neural population. Nature 454:995-999.

Pouzat C, Mazor O, Laurent G (2002) Using noise signature to optimize spike-sorting and to assess neuronal classification quality. J Neurosci Methods 122:43-57.
Prentice JS, Homann J, Simmons KD, Tkačik G, Balasubramanian V, Nelson PC (2011) Fast, scalable, Bayesian spike identification for multielectrode arrays. PLoS One 6:e19884.

Puchalla JL, Schneidman E, Harris RA, Berry MJ (2005) Redundancy in the population code of the retina. Neuron 46:493-504.

Quiroga RQ, Nadasdy Z, Ben-Shaul Y (2004) Unsupervised spike detection and sorting with wavelets and superparamagnetic clustering. Neural Comput 16:1661-1687.

Rinberg D, Bialek W, Davidowitz H, Tishby N (2003) Spike sorting in the frequency domain with overlap detection. arXiv:physics/0306056.

Rochefort NL, Narushima M, Grienberger C, Marandi N, Hill DN, Konnerth A (2011) Development of direction selectivity in mouse cortical neurons. Neuron 71:425-432.

Rust NC, Schwartz O, Movshon JA, Simoncelli EP (2005) Spatiotemporal elements of macaque v1 receptive fields. Neuron 46:945-956.

Sakai HM, Machuca H, Naka KI (1997) Processing of color- and noncolorcoded signals in the gourami retina. II. Amacrine cells. J Neurophysiol 78:2018-2033.

Schneidman E, Berry MJ 2nd, Segev R, Bialek W (2006) Weak pairwise correlations imply strongly correlated network states in a neural population. Nature 440:1007-1012.

Schneidman E, Puchalla JL, Segev R, Harris RA, Bialek W, Berry MJ 2nd (2011) Synergy from silence in a combinatorial neural code. J Neurosci 31:15732-15741.

Schnitzer MJ, Meister M (2003) Multineuronal firing patterns in the signal from eye to brain. Neuron 37:499-511.

Segev R, Goodhouse J, Puchalla J, Berry MJ 2nd (2004) Recording spikes from a large fraction of the ganglion cells in a retinal patch. Nat Neurosci 7:1154-1161.

Segev R, Puchalla J, Berry MJ 2nd (2006) Functional organization of ganglion cells in the salamander retina. J Neurophysiol 95:2277-2292.

Wässle H, Boycott BB (1991) Functional architecture of the mammalian retina. Physiol Rev 71:447-480.

Werblin FS, Dowling JE (1969) Organization of the retina of the mudpuppy, Necturus maculosus. II. Intracellular recording. J Neurophysiol 32: $339-355$. 